\title{
Changes in sprint performance and sagittal plane kinematics after heavy resisted sprint training in professional soccer players
}

\author{
Johan Lahti ${ }^{\text {Corresp., } 1}$, Toni Huuhka ${ }^{2}$, Valentin Romero ${ }^{3}$, Ian Bezodis ${ }^{4}$, Jean-Benoit Morin ${ }^{1,5,6}$, Keijo Häkkinen ${ }^{2}$ \\ 1 LAMHESS, Université de Nice-Sophia Antipolis, Nice, France \\ 2 Neuromuscular Research Center, Biology of Physical Activity, Faculty of Sport and Health Sciences, University of Jyväskylä, Jyvaskyla, Finland \\ ${ }^{3}$ Centre for Sport Studies, Universidad Rey Juan Carlos, Madrid, Spain \\ ${ }^{4}$ Cardiff School of Sport and Health Sciences, Cardiff Metropolian University, Cardiff, United Kingdom \\ 5 UJM-Saint-Etienne Interuniversity Laboratory of Human Movement Biology, Université de Lyon, Saint-Étienne, France \\ 6 Sports Performance Research Institute New Zealand (SPRINZ), Auckland University of Technology, Auckland, New Zealand \\ Corresponding Author: Johan Lahti \\ Email address: johan.lahti1@etu.unice.fr
}

Background. Sprint performance is an essential skill to target within soccer, which can be likely achieved with a variety of methods, including different on-field training options. One such method could be heavy resisted sprint training. However, the effects of such overload on sprint performance and the related kinetic changes are unknown in a professional setting. Another unknown factor is whether violating kinematic specificity via heavy resistance will lead to changes in unloaded sprinting kinematics. We investigated whether heavy resisted sled training (HS) affects sprint performance, kinetics, sagittal plane kinematics, and spatiotemporal parameters in professional male soccer players. Methods. After familiarization, a nine-week training protocol and a two-week taper was completed with sprint performance and force-velocity (FV) profiles compared before and after. Out of the two recruited homogenous soccer teams ( $N=32$, age: $24.1 \pm 5.1$ years: height: $180 \pm$ $10 \mathrm{~cm}$; body-mass: $76.7 \pm 7.7 \mathrm{~kg}, 30$-m split-time: $4.63 \pm 0.13 \mathrm{~s}$ ), one was used as a control group continuing training as normal with no systematic acceleration training (CON, $\mathrm{N}=13$ ), while the intervention team was matched into two HS subgroups based on their sprint performance. Subgroup one trained with a resistance that induced a $60 \%$ velocity decrement from maximal velocity $(\mathrm{N}=10, \mathrm{HS60} \%)$ and subgroup two used a $50 \%$ velocity decrement resistance ( $\mathrm{N}=9$, HS50\%) based on individual load-velocity profiles . Results. Both heavy resistance subgroups improved significantly all 10-30-m split times ( $p<0.05$, $d=-1.25 ;-0.62$ ). Post-hoc analysis showed that HS50\% improved significantly more compared to CON in $0-10$-m split-time $(d=1.03)$ and peak power $(d=1.16)$. Initial maximal theoretical horizontal force capacity and sprint FV-sprint profile properties 
showed a significant moderate relationship with F0 adaptation potential ( $p<0.05)$. No significant differences in sprinting kinematics or spatiotemporal variables were observed that remained under the between-session minimal detectable change. Conclusion. With appropriate coaching, heavy resisted sprint training could be one pragmatic option to assist improvements in sprint performance without adverse changes in sprinting kinematics in professional soccer players. Assessing each player's initial individual sprint FV-profile may assist in predicting adaptation potential. More studies are needed that compare heavy resisted sprinting in randomized conditions. 
1 Changes in sprint performance and sagittal plane kinematics after heavy resisted sprint 2 training in professional soccer players

3

4 Article type: Original article

5 Running head: Heavy resisted sprint training in soccer players

7 Johan Lahti ${ }^{*}$, Toni Huuhka ${ }^{2}$, Valentin Romero ${ }^{3}$, Ian N. Bezodis ${ }^{4}$, Jean-Benoit Morin ${ }^{1,5,6}$, Keijo 8 Häkkinen $^{2}$

${ }^{1}$ Université Cote d'Azur, LAMHESS, Nice, France

$11{ }^{2}$ Neuromuscular Research Center, Biology of Physical Activity, Faculty of Sport and Health Sciences, University of Jyväskylä, Jyväskylä, Finland

${ }^{3}$ Centre for Sport Studies, Rey Juan Carlos University, Madrid, Spain

${ }^{4}$ Cardiff School of Sport and Health Sciences, Cardiff Metropolitan University, Cardiff, UK.

${ }^{5}$ Sports Performance Research Institute New Zealand (SPRINZ), Auckland University of

$17{ }^{6}$ UJM-Saint-Etienne Inter university Laboratory of Human Movement Biology, University of Lyon, Saint-Étienne, France

*Corresponding author:

21 Johan Lahti, MSc

22 Université de Nice Sophia Antipolis - UFR des sciences du sport, 261 Bd du Mercantour, 06200

23 Nice, France

24 Email: lahti.johan87@gmail.com 


\section{Abstract}

Background. Sprint performance is an essential skill to target within soccer, which can be likely achieved with a variety of methods, including different on-field training options. One such method could be heavy resisted sprint training. However, the effects of such overload on sprint performance and the related kinetic changes are unknown in a professional setting. Another unknown factor is whether violating kinematic specificity via heavy resistance will lead to changes in unloaded sprinting kinematics. We investigated whether heavy resisted sled training (HS) affects sprint performance, kinetics, sagittal plane kinematics, and spatiotemporal parameters in professional male soccer players. Methods. After familiarization, a nine-week training protocol and a two-week taper was completed with sprint performance and force-velocity (FV) profiles compared before and after. Out of the two recruited homogenous soccer teams $(\mathrm{N}=32$, age: $24.1 \pm 5.1$ years: height: 180 $\pm 10 \mathrm{~cm}$; body-mass: $76.7 \pm 7.7 \mathrm{~kg}, 30$-m split-time: $4.63 \pm 0.13 \mathrm{~s}$ ), one was used as a control group continuing training as normal with no systematic acceleration training $(\mathrm{CON}$, $\mathrm{N}=13$ ), while the intervention team was matched into two HS subgroups based on their sprint performance. Subgroup one trained with a resistance that induced a $60 \%$ velocity decrement from maximal velocity ( $\mathrm{N}=10, \mathrm{HS} 60 \%)$ and subgroup two used a 50\% velocity decrement resistance ( $\mathrm{N}=9$, HS50\%) based on individual load-velocity profiles. Results. Both heavy resistance subgroups improved significantly all 10-30-m split times $(\mathrm{p}<0.05$, $\mathrm{d}=-1.25 ;-0.62$ ). Post-hoc analysis showed that HS50\% improved significantly more compared to CON in $0-10-\mathrm{m}$ split-time $(\mathrm{d}=1.03)$ and peak power $(\mathrm{d}=1.16)$. Initial maximal theoretical horizontal force capacity and sprint $\mathrm{FV}$-sprint profile properties showed a significant moderate relationship with F0 adaptation potential $(\mathrm{p}<0.05)$. No significant differences in sprinting kinematics or spatiotemporal variables were observed that remained under the between-session minimal detectable change. Conclusion. With appropriate coaching, heavy resisted sprint training could be one pragmatic option to assist improvements in sprint performance without adverse changes in sprinting kinematics in professional soccer players. Assessing each player's initial individual sprint FV-profile may assist in predicting adaptation potential. More studies are needed that compare heavy resisted sprinting in randomized conditions. 
56 Keywords: strength, resistance training, horizontal force, velocity-based training, technique

\section{Introduction}

58

59

60

61

62

63

64

65

66

67

Sprinting performance has been shown to be effective in distinguishing different levels of soccer players ${ }^{1,2}$. Accordingly, it makes sense that there exists an interest in finding optimal methods to improve sprint performance in high level settings ${ }^{1}$. This likely also explains the fact that articles on soccer and sprinting have increased exponentially in the last two decades ${ }^{3}$. However, there still seems to be a lack of sprint performance intervention articles, especially in professional settings. Therefore, researching the usefulness of different training options for sprint performance enhancement within a professional soccer setting seems warranted.

One option that may provide a beneficial stimulus for sprint performance is resisted sprint training 4-12. Different forms of resisted sprint training have been used with the aim to improve sprint performance by overloading different parts of the sprint acceleration phase, both from a intermuscular coordination and structural standpoint ${ }^{12}$. Recently, there has been a growing interest in exploring the value of heavy resistance in assisting improvements in sprint performance ${ }^{6-8}$. Based on the available literature, a definitive definition for heavy resisted sprinting does not seem to exist. One definition for heavy resistance could be that it prioritizes within moderation overloading kinetic properties (force application) over kinematic specificity (technical similarity). Thus, this would be considered "specific traditional overload" ${ }^{13}$. According to cross-sectional biomechanical studies, this corresponds to all loads clearly decreasing maximal velocity capacity more than $10 \%{ }^{14}$. This has also been reported to be around a less accurate measure of $7.5-15 \%$ of body mass (BM), a method that is highly biased towards frictional components and does not consider the relative strength of the athlete ${ }^{15}$. The idea behind heavy loading is to focus on the early acceleration phase of the Force-Velocity (FV) spectrum. Thus from a kinetic standpoint, the focus is on highly overloading the horizontal component of the resultant ground reaction force vector ${ }^{6,16,17}$. This stimulus could affect to different degrees both mechanical effectiveness of the ground force orientation during the step (i.e. what ratio of anterior-posterior and vertical forces is the resultant force built upon) and absolute force output, which could lead to improved sprint performance. 
85

86

87

Interventions with heavy loads have shown mixed results, possibly to some degree due to different methodology. Four studies showed positive effects on early sprint performance ${ }^{4-6,18}$, another showed split time improvements between 10-30-m, while instead a lighter load group improved also at $0-20-\mathrm{m}^{7}$, and one study showed trivial to small effects on performance from both heavy and light resisted sprinting ${ }^{8}$. Evident methodological differences include large differences in what is considered heavy (range $\sim 20 \%-50 \%$ velocity decrement), not standardizing each subjects load to a specific velocity decrement (using the less accurate \% of BM method) ${ }^{19}$, using 1 vs. 2 training sessions per week, initial level and amount of familiarization of subjects, and timing between training completion and post-testing and associated tapering ${ }^{20}$. Limitations have also been discussed, such as not considering each subjects degree of loading needs in terms of initial sprint FV-characteristics in the start of the study ${ }^{8}$.

Furthermore, potential negative effects of violating kinematic specificity by using heavy resistance in sprinting have also been discussed in literature ${ }^{9,10,21}$. These discussions have possibly created uncertainty among coaches, with regards to whether such immediate kinematic and spatiotemporal changes would then lead to detrimental long-term transference to unloaded sprinting. One theory is that training with increased loading may lead to excessive trunk lean ${ }^{10}$, or create a biomechanically less optimal lower body mechanics, such as excessive flexion ${ }^{21}$. However, only two intervention studies have addressed the long-term effects of resisted sprint training on technique and both using only light resistance $(7.5-10 \%$ velocity decrement), while comparing to a unresisted sprint training group ${ }^{10,11}$. Despite the light loading, both interventions showed that resisted sprint training led to a very slight increase in trunk lean during initial acceleration, while one of the studies showed that even the unresisted group increased trunk lean 11. Increased trunk lean has been associated with improved force production in the anteriorposterior direction ${ }^{22}$, thus making it less clear when it is a unwanted adaptation and whether it is dependent on the training modality. Therefore, one possible explanation for why the unresisted group in Alcaraz et al. ${ }^{10}$ did not increase trunk lean could be related to the fact that there was no improvement in early acceleration performance, unlike the unresisted group in Spinks et al. ${ }^{11}$. However, adaptations to kinematics should be carefully interpreted to whether it is a cause or an effect and as such may not be directly related. 
114 Therefore, the aim of this study is to investigate changes in sprint performance and the potential 115 underlying mechanical changes (kinematics, spatiotemporal variables, ground force orientation 116 efficiency, and main kinetic outputs) after integrating two different heavy resisted sprint training 117 loading protocols within a professional soccer setting. The aim of the first heavy load is to follow 118 the same maximal mechanical power parameters as in previous literature, which corresponds to a $11950 \%$ velocity decrement relative to maximal velocity $8,15,29$. The aim of the second heavy load is 120 to have a slightly higher focus on maximal strength and early acceleration, which corresponds to 121 a $60 \%$ velocity decrement. Our first hypothesis was that both heavy loads will improve early split122 time sprint performance, with the heavier load being even more effective at early acceleration. Our 123 second hypothesis was that both loads will increase early acceleration center of mass (CM) 124 distance and CM angle at toe-off.

\section{Materials and methods}

\section{Study design and participants}

130

131

132

133

A pre-test versus post-test experimental design utilizing three groups was used to examine the effects of heavy resisted sprint training in professional male soccer players. 32 male professional soccer athletes from two teams in the premier division in Finland, volunteered to participate in the study using convenience sampling (age: $24.1 \pm 5.1$ years: body-height: $180 \pm 10 \mathrm{~cm}$, body-mass: $76.7 \pm 7.7 \mathrm{~kg}$ ). The sample size in this study was highly similar to previous resisted sprint training studies using comparable methods ${ }^{10,11}$. Inclusion criteria included being a professional soccer athlete competing within the Finnish Premier soccer league. An exclusion criterion was placed for goalkeepers due to the lower amounts of linear sprinting. No exclusion criterion was placed for age, but under 18-year-old athletes were required to have parental consent. Both teams were in initial pre-season and trained on average of 7-10 sessions per week (which included strength training twice per week) and competed an average of once per week. More detailed scheduling can be found in the supporting information (tables 1-2 in S2 tables). One professional soccer team was used as two intervention groups and the other professional soccer team as a control group. The soccer team selected to function as the control group did not train early or late acceleration 
144 separately from sport-specific practice in their pre-season protocol, including no resisted sled 145 training. Therefore, they were instructed to continue training as normal. The intervention team was 146 further randomly matched into two homogenous subgroups in terms of sprint performance with 147 different heavy sled loading schemes. These loading schemes corresponded either to a heavy sled 148 (HS) load that decreased the athlete's maximal velocity by 50\% (HS50\%) or 60\% (HS60\%). A 149 total of 15 training opportunities were provided within 9 weeks (Fig 1). Including two training 150 sessions each week was not possible because of the teams scheduling conflicts. This corresponded 151 to 6 out of 9 weeks including two sessions per week. Furthermore, tapering was initiated on week 15210 and continued to week 11 where post testing was performed. Therefore, both the control and 153 intervention group were tested for sprint performance and kinematic changes 11 weeks apart. 154 Testing was performed on the same day of the week (end of the week, after a low intensity day), 155 but one week apart. The intervention groups had the opportunity to complete two weeks of 156 pretesting on sprint performance and technique analysis, while due to scheduling issues, the control 157 group was available for one week of testing. All training and testing sessions were completed 158 inside on artificial turf, with an exception made for post testing, which was performed outside on 159 the same type of artificial turf on the same time and day of the week. Wind conditions were still $160\left(1 \mathrm{~m} . \mathrm{s}^{-1}\right)$ on the outdoor post testing day with a highly similar temperature (14 vs. $\left.15 \mathrm{C}\right)$. Written 161 informed consent was obtained from all athletes on the first day of familiarization, and approval 162 for this study was granted by the University of Jyvaskyla Ethical Committee and was performed 163 in the accordance with the Declaration of Helsinki.

164

165

166

167

168

169

170

171

172

173

\section{Insert figure 1 here}

\section{Group allocation}

Athletes in the intervention soccer team were ordered from the lowest to highest $30-\mathrm{m}$ split times derived during two weeks of familiarization and, thereafter, matched in a pairwise manner into either of the following heavy sled groups: HS50\% or HS60\% to balance variance. The best 30-m performance was used from the two familiarization weeks. The 0-30-m split time was used as it has a lower measurement error compared to smaller split-times ${ }^{23}$, and because it was the

Peer) reviewing PDF | (2020:07:51064:2:0:NEW 5 Nov 2020) 
174 maximal split-time distance used in our testing protocol. There was no ordering of the control

175 group, however, the sprint performance was predicted to be similar due to earlier research

176 collaboration work with the team involving sprint performance testing. The initial aim was to

177 recruit an equal amount of soccer athletes within the control team. However, only 13 were

178 available to volunteer and were considered healthy by the team physiotherapist to perform sprint

179 testing at this point of the early pre-season. The final group size and respective highly

180 homogenous 30-m performance times were the following: HS60\%, $\mathrm{N}=10,4.65 \mathrm{~s}, \mathrm{CI} 95 \%$ : 4.55;

1814.77 vs. HS50\%, N = 9, 4.62 s, CI95\%: 4.56; 4.69 vs. CON, N: 13, 4.63 s, CI95\%: 4.55; 4.70, p

$182=0.88$.

183

\section{Testing procedures and data analysis}

185

\section{Sprint Force-Velocity profile and performance tests}

187 Following warm-up, all participants completed two 30-m maximal sprints from a standing stance start. The passive recovery between sprints was three minutes. Sprint performance (split times 05,0-10,0-20, and 0-30-m), kinetic outputs and mechanical efficiency were computed pre- and posttraining from the best time trial. Data was derived from a radar device (Stalker ATS Pro II, Applied Concepts, TX, USA), using a validated field method as reported previously ${ }^{23-25}$. Individual linear sprint Force-Velocity (FV) profiles in the antero-posterior direction were calculated and thereafter relative theoretical maximal force $\left(F 0: \mathrm{N} . \mathrm{kg}^{-1}\right)$, velocity $\left(v 0: \mathrm{m}_{\mathrm{s}} \mathrm{s}^{-1}\right)$, and maximal power (Pmax: $W . \mathrm{kg}^{-1}$ ) capabilities. Despite the use of an approximate measurement of "maximal power", which can be considered a pseudo-power ${ }^{26}$, the term maximal power output will be used in this study. Mechanical efficiency was calculated based on the maximal ratio of forces (RFmax in \%) and the average ratio of forces for the first 10-m (Mean RF on 10-m in \%). These RF values are a ratio of the step-averaged horizontal component of the ground-reaction force to the corresponding resultant force, i.e. these values aid the interpretation of mechanical effectiveness with which the ground force is oriented in early acceleration ${ }^{27}$. RFmax depicts the theoretical maximal effectiveness of directing force forwards in the first step of the sprint (within the constraints of sprint running stance, the higher the value of RFmax, the more forward, horizontally-oriented the ground push 
204 the forward force application effectiveness over the first 10-m. A more horizontally oriented

205 ground reaction force was considered beneficial within the range of values reported in this study.

\section{Load-velocity tests}

208 The final sled familiarization session was combined with load-velocity testing. Load-velocity tests

209

210

211

212

213

214

215

216

217

218

219

220

221

222

223

224

225

226

227

228

229

230 were completed using one unresisted and 3 resisted sprints $(50 \%, 75 \%, 100 \%$ of BM) for both HS groups, outlined in previous literature ${ }^{28}$. Thereafter, individualized load-velocity profiles were created for each athlete with a least-square linear regression ${ }^{28}$. The individual resistance leading to a $60 \%$ and $50 \%$-velocity decrement from maximal velocity was calculated.

Sled velocity was verified with the radar on the first week of training to be within a $5 \%$ range of the targeted velocity. A total of 3 athletes' loads had to be modified with an increase of $2.5-7.5 \mathrm{~kg}$, that were verified again the following week (Final ranges, HS\%60: -58.4\%, CI95\%: -59.4; -57.5, HS50\%: -49.4\%, CI95\%: -51.4; -47.5).

\section{Sprint spatiotemporal and kinematics assessment}

For all FV-profile sprints, video images were obtained at $240 \mathrm{~Hz}$ with a smart phone video camera at a HD resolution of 720p (Iphone6, Apple Inc, Cupertino, Ca). The kinematic sprint sequences of interest were the touchdown (first frame the foot was visibly in contact with the ground) and toe-off (first frame the foot had visibly left the ground) across the first extension and three steps of early acceleration and 3 steps in upright sprinting of the sprint using $6 \times$ zoom in Kinovea (v.0.8.15), similar to previous literature ${ }^{29}$. The same leg sequence was analyzed pre-post, with a secondary effort to analyze the sequence as close to the midpoint of the camera as possible. The cameras were placed 9-m perpendicular at the 1.5-m mark and the 22.5-m mark along a 0-30-m line, at a $1.1 \mathrm{~m}$ height, allowing approximately a 9-m field of view. 1.5-m was chosen based on that the first three steps have been considered unique to early acceleration ${ }^{30}$, taking place within 
231 around three meters in this population. Upright mechanics were analyzed at $22.5-\mathrm{m}$ based on that

232 team sport athletes are at around $95 \%$ or at maximal velocity at this phase ${ }^{31}$.

233 Furthermore, an additional data analysis was performed in the second week of the study to observe

234 the immediate effects of the resisted sprint training on early acceleration mechanics. The second

235 week was chosen so that the athletes had time to react to the used coaching cues, which are defined

236 in the intervention section. According to our data, sleds at this resistance magnitude reach maximal

237 velocity around 5-m, therefore going into a velocity maintenance phase for the remaining meters

238 ( $\sim 10$-m for HS60\%, 15-m for HS50\%). Thus, this was considered the main stimuli zone for each

239 sprint, and therefore, it was used to compare to early acceleration of the unloaded sprint. This was

240 done by having the sled sprint start 5-m before the calibration zone for unloaded early acceleration.

241 All filming zones were calibrated to a 5-m horizontal distance along the midpoint of the camera at

242 the line. The human body was modelled as 18 points. This required manual digitization of the

243 following: vertex of the head, halfway between the suprasternal notch and the $7^{\text {th }}$ cervical vertebra,

244 shoulder, elbow, and wrist joint centers, head of third metacarpal, hip, knee, and ankle joint centers,

245 and the tip of the toe.

246 The following spatiotemporal and kinematic step characteristics were determined after exporting 247 the digitalized coordinates to Excel (Microsoft Office 2016): contact time (s), step length (m; 248 horizontal displacement between initial contact of one foot and the point of initial contact of the 249 opposite foot, measured from the toe tips), and step rate (Hz; calculated as 1/step time, where step 250 time was determined as the sum of contact time and the subsequent aerial time). Whole-body center 251 of mass (CM) location was calculated using de Leva's et al. ${ }^{32}$ segmental data. This allowed for 252 the calculation of touchdown and toe-off distances (horizontal distance between the toe and the $253 \mathrm{CM}$, with positive values representing the toe ahead of the CM). Furthermore, angles of the trunk 254 (relative to the horizontal) and the hips (ipsilateral and contralateral) were quantified. All distances 255 of CM were normalized to the height of the athlete and reported as (m/body length) ${ }^{29}$. All sprints 256 were analyzed twice to improve reliability with the digital marker method. 
260 Training protocols are outlined in Figure 1. Familiarization within the intervention group for sled

261 training was initiated two weeks before the training intervention and was combined with the sprint

262 Force-Velocity (FV)-profile tests (2x30-m sprints), including group allocation based on sprint 263 performance. A load of $80 \%$ of BM ( $2 \times 15-\mathrm{m}$ sprints $)$ was selected for familiarization. A total of 26415 heavy resisted sprint training session opportunities were planned within 9 weeks and an 265 additional two-week taper (two sessions total) across the 11-week pre-season. This 11-week 266 interval included a break week in the form of an international training camp. Therefore, resisted 267 sprint training sessions were, in general, twice per week, transitioning from a total of six resisted 268 sprints per week up to eight at the midway point (week 5). All training sessions included 20-m free 269 sprints, which were in the start of the program two per session, transitioning to one free sprint per 270 session after the midway point. All athletes were harnessed at their waist, using the $21 \mathrm{~kg}$ sprint 271 sleds (DINOX, customized sled, Finland). To standardize the stimuli between athletes within both 272 intervention subgroups, a velocity-based training approach was utilized, where all athletes used a 273 load that adapted their velocity to the desired threshold. In this case HS60\% used a load leading to 274 a $60 \%$ velocity decrement from maximal velocity and HS50\% used a load leading to a 50\% 275 decrement from maximal velocity. The 50\% load was chosen to simulate power properties as it 276 has been shown that external maximal power is reached approximately at $50 \%$ of maximal velocity 277 in a maximal acceleration sprint ${ }^{28}$. The heavier $60 \%$ velocity decrement load was chosen with the 278 aim to stay within proximity to the $50 \%$ load but stimulate more maximal strength properties, thus 279 an even higher bias towards early acceleration. On the artificial training surface, this 10\% velocity 280 difference corresponded to the average relative mass of $120 \%$ of BM in the HS60\% group and $28194 \%$ of BM in the HS50\% group (including the mass of the sled), equating to a group average 282 difference of $26 \mathrm{~kg}$. A sled sprint distance of $0-15-\mathrm{m}$ for the HS60\% group and 0-20-m for the 283 HS50\% group was used to standardize sprint time (HS60\%: 4.26 s, CI95\%: 3.74; 4.77, HS50\%: $4.73 \mathrm{~s}, \mathrm{CI95 \%}$ : 4.39; 5.08, $\mathrm{p}=0.15)$. Training was supervised by the team strength and 285 conditioning coach and completed after the warm-up for technical and/or tactical training on field. Pre-training warm-up ( 15min) included light running, dynamic full-body stretches, muscle and dynamic movement pattern activation, and low to high intensity sprint exercises. Between-sprint rest was three minutes. Both groups were given the same coaching cues, that is, prioritizing stride power (or push) over stride frequency and high arm movement with aligned posture. Finally, post 
290 testing was completed at the end of a two-week tapering period, by reducing the modality specific

291 volume down from eight sprints a week to two, with one session of two free sprints per week.

\section{Statistical analysis}

293

294

295

296

297

298

299

300

301

302

303

304

305

306

307

308

309

310

311

312

313

314

315

316

317

318

319

Shapiro-Wilk's test was used to test the data's normality and levene's test was used to examine the homogeneity of variance. A $3 \times 2$ (group $\times$ time) repeated-measured ANOVA with Bonferroni post-hoc comparisons was used to determine the within- and between-group effects as well as examining interaction effects. Baseline measures were used as covariates to control for the effect of initial sprint performance. Sprint performance was defined mechanically (Pmax, F0, RFmax, Mean RF on 10-m, v0, and Sprint FV-profile), by split-times (5-m,10-m,20-m, and 30-m), spatiotemporally (contact time, step rate, step length at initial acceleration and maximal velocity) and kinematically (hip angle, trunk angle, CM distance). For each individual the sprint with the best 30-m time within pre and post testing was compared statistically for both mechanical-, split times- and sprint technique variables. Independent and paired two-tailed t-tests were used to analyse within- and between-group differences of the immediate effects of the resisted sprint training on early acceleration mechanics (two groups). Given the large number of analyses (26), we adjusted for multiple comparisons using the Benjamini-Hochberg procedure utilizing a false discovery rate of $0.05^{33}$. Effect sizes (ES) were calculated using pooled SD and interpreted with Hopkins' benchmarks to distinguish small $(\geq 0.2)$, moderate $(\geq 0.6)$, large $(\geq 1.2)$ effects ${ }^{34}$. Accounting for typical fluctuations in athletes' weekly sprint performance and sprint technique was of interest in our study. Thus, minimum detectable change (MDC) with $95 \%$ confidence intervals was calculated from the difference in best performance sprint FV-profile variables completed during pre-test week -1 and $0{ }^{39}$. The sprint with the best $30-\mathrm{m}$ time was used for kinematic and spatiotemporal variables. MDC was derived using Typical Error (TE) • $1.96 \sqrt{ } 2$, and $\mathrm{MDC} \%$ was defined as $(\mathrm{MDC} / \overline{\mathrm{X}}) \cdot 100$. Test-retest reliability for each variable analyzed was assessed by intraclass correlation coefficient $\left(\mathrm{ICC}_{3,1}\right)$, coefficient of variation $(\mathrm{CV} \%)$, TE with 95\% confidence intervals, and MDC, using Hopkins spreadsheet ${ }^{35}$. ICCs were defined as poor (ICC < 0.40), fair $(0.40 \leq \mathrm{ICC}<0.60)$, good $(0.60 \leq \mathrm{ICC}<0.75)$, and excellent $(0.75 \leq \mathrm{ICC} \leq$ 1.00). Alpha was set at $\mathrm{p}<0.05$. Descriptive data are presented as mean \pm standard deviation (SD). 
320

321

322

323

324

325

326

327

328

329

330

331

332

333

334

335

336

337

338

339

340

341

342

343

344

345

346

347

\section{Results}

A total of four subjects could not complete the required pre-post measurements. Due to sustaining a flu, one athlete within the HS60\% group could not perform final testing, making a total of nine out of 10 subjects completing the protocol. Due to injuries, three subjects in the control group could not participate in the post testing, making a total of 10 subjects measured. Furthermore, although participating in the sprint performance measurements, there was one camera malfunction during the HS50\% group post-testing, leading to a loss of pre-post kinematics of one subject.

Out of 15 possible sessions, within the 9-week window the HS60\% completed an average of 10.6 (CI95\%: 9.57; 11.54), while HS50\% completed an average of 10.3 (CI95\%: 9.30; 11.37). For HS60\%, this corresponded to a resisted sprint volume of 38.2 (CI95\%: 35.5; 40.9) and for HS50\% 37.4 (CI95\%: 34.2; 40.7), $\mathrm{p}=0.72$.

\section{Group Characteristics at Baseline}

All variables were normally distributed. For the final sample completing the study, baseline population variance was not significantly different for any variables, including age, height, mass, kinetic and kinematic variables $(\mathrm{p}>0.09)$, with all split-times being highly similar (Table $1, \mathrm{p}>$ $0.55)$.

\section{Reliability}

All reliability statistical values can be found in supporting information (tables 1-8 in S1 tables), including MDC\%, TE, CV\% and ICC. For the sprint FV-profile and performance variables, within and between session ICC ranged from good to excellent (0.60-0.98, CI95\%: -0.09; 0.99), except for sprint FV-profile slope and mean RF on 10-m, with RF on 10-m showing poor between session reliability (0.23, CI95\%: -0.57 ; 0.81), and FV-profile slope showing fair reliability $(0.49$, CI95\%: $-0.33 ; 0.89$ ). For the reliability of the digitization process (within sprint spatiotemporal and kinematic variables), ICC was excellent $(0.83-0.99$, CI95\%: 0.38; 0.99). For the within and 
348 between session spatiotemporal and kinematic variables, ICC ranged from fair to excellent $(0.41$ $349-0.99$, CI95\%: 0.03; 0.99), except for maximal velocity contact time, showing poor within-session 350 reliability $(0.34, \mathrm{CI}:-0.37 ; 0.80)$.

351

352

353

354

355

356

357

358

359

360

361

362

363

364

365

366

367

368

369

370

371

372

373

374

\section{Between and within group statistics}

\section{Body mass}

No significant differences were found at baseline and pre and post for BM in the 3 groups ( $\mathrm{p}>$ $0.05)$.

\section{Sprint Split-times}

All descriptive and inferential statistics for sprint performance can be found in table 1 and visualized in Figure 2. All split-times showed significant main effects for time $(\mathrm{p}<0.05)$. Posthoc analyses revealed significant improvements in both $\mathrm{HS} 60 \%$ and $\mathrm{HS} 50 \%$ for $10-\mathrm{m}$ $(\mathrm{HS} 60 \%, \mathrm{p}=0.001, \mathrm{~d}=-0.96 ; \mathrm{HS} 50 \%, \mathrm{p}<0.001, \mathrm{~d}=-1.25), 20-\mathrm{m}(\mathrm{HS} 60 \%, \mathrm{p}=0.008, \mathrm{~d}=-$ 0.77; HS50\%, p $<0.001, \mathrm{~d}=-1.15$ ), and 30-m split-times (HS60\%, p = 0.02, $\mathrm{d}=-0.62$; $\mathrm{HS} 50 \%, \mathrm{p}<0.001, \mathrm{~d}=-1.18$ ) after controlling for baseline performance. HS50\% was the only group to significantly improve $5-\mathrm{m}$ split-time $(\mathrm{p}=0.005, \mathrm{~d}=-1.07)$, although a trend was present for HS60\% ( $\mathrm{p}=0.05, \mathrm{~d}=-0.74)$. However, only 0-10-m, 0-20-m, and 0-30-m split time improvements surpassed the between-session minimal detectable change threshold (Figure 2). This means that the changes in 5-m split-times could be due to normal weekly fluctuations in performance combined with measurement error. A group $\times$ time interaction effect was observed for 10 -m split-time $(F(2,24)=4.031, \mathrm{p}=0.031)$. Post-hoc analysis revealed that 10 -m split-time improved significantly more in HS50\% compared to CON over the study period $(\mathrm{p}=0.03, \mathrm{~d}=$ $1.03)$. 


\section{Sprint Force-Velocity profile variables}

380

381

382

383

384

385

386

387

388

389

390

391

392

393

394

395

396

397

398

All within- and between-group statistics for mechanical variables can be found in table 2 . Correlations between mechanical variables can be found in Figure 3. All mechanical variables showed significant main effects for time $(\mathrm{p}<0.05)$. Post-hoc analyses revealed significant improvements in both HS60\% and HS50\% for F0 (HS60\%, p = 0.02, d = 1.00; HS50\%, p = 0.002, $\mathrm{d}=1.04)$, Mean RF on 10-m (HS60\%, $\mathrm{p}=0.013, \mathrm{~d}=0.80 ; \mathrm{HS} 50 \%, \mathrm{p}<0.001, \mathrm{~d}=1.14)$, and Pmax (HS60\%, p $=0.011, d=0.84 ; \mathrm{HS} 50 \%, \mathrm{p}<0.001, \mathrm{~d}=1.18)$ after controlling for baseline values. However, the F0 changes (HS60\%: $7.83 \%$, HS50\%: $9.23 \%$ ) were under the betweensession minimal detectable change threshold (9.53\%). RFmax improved significantly in all groups $(\mathrm{HS} 60 \%, \mathrm{p}=0.011, \mathrm{~d}=1.25 ; \mathrm{HS} 50 \%, \mathrm{p}=0.001, \mathrm{~d}=1.01 ; \mathrm{CON}, \mathrm{p}=0.041, \mathrm{~d}=0.55)$. There was a significant improvement in $\operatorname{HS} 50 \%$ for $v 0(\Delta 3.08 \%, \mathrm{p}=0.04, \mathrm{~d}=0.78)$, however, the result remained under the between session minimal detectable change threshold $(3.13 \%)$. No other within-group significant changes were observed $(\mathrm{p}>0.05)$. A group $\times$ time interaction effect was observed for Pmax $(\mathrm{F}(2,24)=4.055, \mathrm{p}=0.030)$, and a trend for $\mathrm{F} 0(F(2,24)=2.778, \mathrm{p}=0.082)$. Post-hoc analysis revealed that Pmax improved significantly more in HS50\% compared to CON over the study period $(\mathrm{p}=0.03, \mathrm{~d}=1.16)$. No other between-group differences were observed. 
399

400

401

402

403

404

405

406

407

408

409

410

411

412

413

414

415

416

417

418

419

420

421

422

423

424

425

\section{Sprint kinematic and spatiotemporal variables}

\section{Cross-sectional analysis of immediate effects of sled on early acceleration}

All significant results for immediate effects of sled are visualized in Figure 4. All descriptive and inferential statistics can be found in table 3. Due to timetable issues, 8 out 9 subjects were available for kinematic filming of the sled from the HS60\% group and 6 out of 9 from the HS50\% group.

Between-group t-tests showed no differences $(\mathrm{p}>0.05)$. Within group t-test comparisons with Benjamini-Hochberg corrections showed that the provided resistance from the sled led to significant changes in both spatiotemporal and kinematic variables. All spatiotemporal variables changed significantly in the HS60\% group, with increased contact time $(\mathrm{p}=0.003, \mathrm{~d}=2.10)$, step rate $(p=0.004, d=-1.90)$, and step length $(p=0.008: d=-1.58)$. Both sled loads significantly decreased touchdown CM distance (HS60\%: $p=0.003, d=1.99 ;$ HS50\%: $p=0.003, d=3.50$ ) and $\mathrm{CM}$ angle at touchdown (HS60\%: $\mathrm{p}=0.005, \mathrm{~d}=-2.30, \mathrm{HS} 50 \%: \mathrm{p}=0.005, \mathrm{~d}=-3.00$ ), corresponding to taking steps further behind center of mass. No other variables reached significance $(\mathrm{p}>0.05)$.

4

5

Insert figure 4 here

Insert table 3 here

\section{Pre-Post intervention changes in kinematic and spatiotemporal variables}

All descriptive and inferential statistics for sprint technique can be found in table 4 and visualized in Figure 5. In early acceleration, there was a main effect for time in step length, contralateral hip angle at toe-off, and contralateral hip angle at touchdown. At maximal velocity, there was a main effect for time in step rate, trunk angle at toe-off, hip contralateral angle at toeoff, and CM angle at toe-off. Post-hoc analyses revealed a significant decrease in both HS60\% and CON for contralateral hip angle at touchdown during early acceleration (HS60\%: $\Delta-4.01$ 
$426 \%, \mathrm{p}=0.004, \mathrm{~d}=-0.80 ; \mathrm{CON}: \Delta-3.13 \%, \mathrm{p}=0.006, \mathrm{~d}=-0.80)$ after controlling for baseline

427 values. However, the result remained under the between session minimal detectable change

428 threshold (5.85\%). All other within-group comparisons did not reach significance $(\mathrm{p}>0.05)$.

429 No interaction effects were found for pre and post sprint kinematic och spatiotemporal variables 430 for both early acceleration and upright sprinting $(\mathrm{p}>0.05)$.

431

432

Insert figure 5 here

433

Insert table 4 here 


\section{Discussion}

435

436 The main results of this study were that, although both heavy load conditions (50\% and 60\%

437 velocity decrement) improved sprint performance in soccer players, the HS50\% was the only 438 group showing changes in sprint parameters that were significantly different from CON. A clear favoring towards improvements in early acceleration performance and sprint kinetics were present in both $\mathrm{HS} 50 \%$ and HS60\% groups, showing moderate to large effect size differences compared to CON. Furthermore, although both loads produced significant immediate changes in early acceleration at toe-off and touchdown, no long-term changes on early acceleration and upright sprint technique were observed that surpassed the minimal detectable change. These results suggest that heavy resisted sprinting can be successfully integrated in a professional soccer setting, potentially preferably with resistance associated to a $\sim 50 \%$ drop in maximal running velocity compared to $\sim 60 \%$.

447 Our initial hypothesis was partly met, with heavy resisted sprinting leading to improved early acceleration sprint performance. It is important to mention that the reported 5-m within-group improvements fell under the minimal detectable change threshold and, thus, still could be interpreted as remaining within the measurement error thresholds (Figure 2). This is a logical result based on previous literature on 5-m split time measurements ${ }^{36}$. However, we expected to see differences between the heavy loads in improving specific parts of early acceleration sprint performance. Specifically, we expected the HS60\% group to mostly improve the $0-5-\mathrm{m}$ splittimes, whereas the HS50\% group would mostly improve the $0-10-\mathrm{m}$ split times. This is because the first steps of acceleration are considered to be more dependent on maximal force capacity, with its importance reducing with increasing velocity ${ }^{17,37}$. Hence the larger load was thought to provide a higher transfer in this area. However, both heavy loads had similar effects on early acceleration performance (Figure 2). Although the HS50\% group was the only group to reach significantly lower split-times compared to CON and had a large effect size (0-10-m split-time). Furthermore, Figure 2 shows trends towards HS50\% providing a broader stimulus across the entire acceleration phase. Future studies should verify how reproducible this adaptation signature 
463 small difference in loading parameters and that the total training volume was possibly not high 464 enough.

465 The underlying kinetic reasons to the performance improvements were also of interest in this 466 study. Therefore, we analyzed the ratio of forces at the first step and over the first 10-m (RFmax 467 and mean RF on 10-m). However, caution should be considered within the interpretation of mean 468 RF on 10-m, showing poor between-session reliability within this population. The analysis 469 showed that when considering initial values, there was a lack of clear difference in effect size 470 between RFmax and F0 compared to the control group (both moderate effects). Therefore, 471 improvements in both their maximal ground reaction force capacity and their capability to orient 472 this force more horizontally may have contributed to improved sprint performance. However, 473 Pmax was the only biomechanical variable to show significant improvements compared to CON, 474 specifically in the HS50\% group. As external maximal power is produced at approximately $50 \%$ 475 of maximal velocity in a maximal acceleration sprint ${ }^{28}$, it makes sense that Pmax was 476 maximized in the HS50\% group. Therefore, the ability to produce higher forces at higher 477 velocities (i.e. maximal mechanical power), seemed to be the main driver for the improved sprint 478 performance.

479 The most important aim of improving sprint performance was met, an essential part in preparing 480 soccer athletes for the season ${ }^{1,2}$. This contradicted previous literature with similar loading 481 parameters. Specifically, the main methodological strengths of this study compared to previous literature was that the present groups were evenly divided based on their initial sprint performance, training was done mostly 1-2 per week instead of once, and tapering was completed ${ }^{7,8}$. Furthermore, in the study by Pareja-Blanco et al. ${ }^{7}$ loads were not standardized and individualized to a specific velocity decrement, but rather to body mass ( $80 \%$ of BM). Therefore, one conclusion is that if a time slot of roughly 20 minutes is accepted for velocity-based resisted sprint training within field practice conditions 1-2 per week, it will likely be beneficial, assuming the athlete has been assessed for lacking early acceleration capacity (Figure 4). However, our study did not have a group completing non-resisted sprint training, only a control group completing sport-specific training. Therefore, we do not know if just the mere systematic focus on early acceleration, regardless of load, is enough. Measuring a force-velocity and load-velocity 492 profile for everyone might be an issue for some as there may be time constraints and lack of 
493 access to technology. However, this can be done relatively quickly and at a low cost with the

494 help of accurate apps ${ }^{38}$, while saving some time with a shorter load-velocity protocol (3 loads: 0 ,

49525 and $75 \%$ of BM is sufficient to obtain the linear individual load-velocity profile, see Figure 2

496 in Cross et al. ${ }^{8}$ ), although this still needs to be validated.

497 Our second hypothesis was that both loads would improve early acceleration toe-off CM distance

498 (more triple extension of the body) and CM angle (increased forward body lean). The results 499 showed no changes in the kinematics or any other variables in early acceleration, which is in 500 contrast to previous light load literature showing slight increases in trunk lean 10,11. However, 501 moderate effect sizes were seen in some early acceleration kinematic parameters, including 502 decreased touchdown CM distance and CM angle in HS50\%, corresponding to potentially less 503 time spent in the breaking phase due to contact times not changing. These changes make sense 504 with our cross-sectional sled measurements (Figure 4), as these were the two variables that showed 505 the largest effect sizes for changes in movement. However, we found no relationships between 506 changes in these variables and improvements in sprint performance, thus more accurate 507 methodological approaches and/or larger sample sizes are likely needed for such short 508 interventions. Furthermore, no negative effects of heavy resisted sprinting were observed on either 509 early acceleration or upright sagittal plane sprint kinematics as speculated to some degree by 510 previous literature $9,10,14,21$. While both $\mathrm{HS} 60 \%$ and CON significantly decreased their contralateral 511 hip angle at touchdown during early acceleration, this was likely due to normal fluctuations in 512 sprint technique as the result remained under the minimal detectable change (HS60: 3.13 \%, CON: $5134.01 \%$, MDC: $5.85 \%$ ), rather than longitudinal alterations caused by the training protocols. One 514 clear explanation is that potential deleterious effects were mitigated via coaching cues targeted to 515 maintain good posture, in place of athletes adopting sub-optimal patterning during the heavy 516 resisted sprinting. Our results cannot support the occurrence of longitudinal technical breakdown 517 following heavy resisted sprint training, or at least indicate that such effects might be reduced with 518 common-sense programming.

519 As an additional observation, our data showed that initial F0 capacity and sprint FV-profile 520 orientation seems to explain moderately adaptation potential (Fig 4), corresponding to previous 521 literature ${ }^{39}$. Thus, if an athlete already has a high force production capacity, or a force-oriented 522 FV-relationship/profile, it should logically reduce adaptation potential to a high force - low 
523 velocity stimulus. This sample size does not allow for clear cut-off thresholds for training,

524 however, a recent study using heavy resisted sprints in high-level rugby players showed nearly

525 identical results. Therefore, an initial F0 value around $8.4 \mathrm{~N} . \mathrm{kg}^{-1}$, or a sprint FV-profile lower than

$526-0.95$ will likely not respond well to heavy resisted sprint training ${ }^{39}$. Future studies should explore

527 if varying from individualized (velocity decrement) heavy to light loads based on initial FV528 qualities is of further value.

\section{Limitations}

530 The control group and the intervention groups were two different teams with inevitable differences 531 in their training culture. Therefore, although initial sprint performance was highly homogenous, 532 differences in training and recovery methods may have contributed to the results. This study also 533 may have been underpowered for some variables, as based on the within- and between-group effect 534 sizes, both groups showed similar trends in early acceleration, but only HS50\% reached statistical 535 significance. Furthermore, inclusion of a randomized control group that performs unloaded 536 systematic acceleration training should be compared in future studies. The 2D motion analysis was 537 only based on two time points, therefore caution is advised in their interpretation and future studies 538 are implored to use more rigorous approaches. We did not have access to a high-resolution slow539 motion camera, which likely contributed a couple of variables showing lower reliability. Similar 540 to previous resisted sled training literature our sled study used a single time point method (toe-off, 541 touchdown). A more ideal approach would likely be the analysis of waveforms, such as with the 542 statistical parametric mapping method ${ }^{40}$. We also acknowledge that the absolute reliability (ICC) 543 confidence intervals can be considered large in numerous analysed variables, making it too 544 imprecise to make accurate conclusions regarding their true reliability. Future studies using similar 545 methods should include a larger sample size to improve reliability measurements.

\section{Conclusion}

549 Providing efficient evidence-based options to enhance sprint performance training is crucial for 550 strength and conditioning coaches in high level soccer settings. It seems that in a time span of 11 551 weeks, one of the underlying reasons for heavy resisted sprint training improving sprint 552 performance is increased force production (both directional and absolute). As this took place in a 
553 similar step time, the main driver seems to be improved mechanical power and likely rate of force

554 development. Thus, our findings suggest that heavy resisted sprint training can improve sprint

555 performance in professional soccer players. Adaptations may be potentially maximized with a 50\%

556 compared to a $60 \%$ velocity drop resistance. A 50\% velocity drop resistance may provide a

557 broader transfer across split-times, which should be verified in future studies. Based on the average

558 amount of resisted sprints that were conducted during this study, the target should be to achieve at

559 least 38 sprints divided over 2 months, preferably 1-2 per week, including a final taper. After

560 familiarization, this stimulus can be integrated efficiently into field conditions, with a session

561 duration lasting $\sim 20$ minutes for the entire team with 4+ sleds. Our results support the assertion

562 that coaches do not have to worry about potential adverse effects on sprint technique if appropriate

563 familiarization, cueing and supervision is used. Furthermore, coaches should be aware that heavy

564 resisted sprint training will very likely not work for the entire team, which can be to some extent

565 predicated by appropriate initial performance tests, including sprint FV-profiling.

\section{Acknowledgements}

568

569 The authors would like to thank all the athletes and coaching staff that were involved in the study.

570 We would also like to thank the following sports scientists for their consultation to the project and

571 aiding in creating the figures; Andrew Vigotsky and James Wild. None of the authors received any

572 commercial funding or salary in any form that would influence the study design, data collection

573 and analysis, decision to publish, or preparation of the manuscript. No other conflicts are present. 
1. Haugen TA, Tønnessen E, Hisdal J, Seiler S. The Role and Development of Sprinting Speed in Soccer. Int J $586 \quad$ Sports Physiol Perform. 2014;9(3):432-441.

587 2. Cometti G, Maffiuletti NA, Pousson M, Chatard J-C, Maffulli N. Isokinetic Strength and Anaerobic Power of Elite, Subelite and Amateur French Soccer Players. Int J Sports Med. 2001;22(1):45-51.

3. Nikolaidis P, Knechtle B, Clemente F, Torres-Luque G. Reference values for the sprint performance in male football players aged from 9-35 years. Biomed Hum Kinet. 2016;8.

591 4. Kawamori N, Newton RU, Hori N, Nosaka K. Effects of Weighted Sled Towing With Heavy Versus Light Load on Sprint Acceleration Ability. J Strength Cond Res. 2014;28(10):2738-2745.

5. Bachero-Mena B, González-Badillo JJ. Effects of Resisted Sprint Training on Acceleration With Three Different Loads Accounting for 5, 12.5, and 20\% of Body Mass. J Strength Cond Res. 2014;28(10):29542960.

596 6. Morin J-B, Petrakos G, Jiménez-Reyes P, Brown SR, Samozino P, Cross MR. Very-Heavy Sled Training for Improving Horizontal-Force Output in Soccer Players. Int J Sports Physiol Perform. 2017;12(6):840-844. 
600 8. Cross MR, Lahti J, Brown SR, Chedati M, Jimenez-Reyes P, Samozino P, Eriksrud O, Morin JB. Training at maximal power in resisted sprinting: Optimal load determination methodology and pilot results in team

602 sport athletes. Sandbakk Ø, ed. PLoS One. 2018;13(4):e0195477.

603

9. Alcaraz PE, Carlos-Vivas J, Oponjuru BO, Martínez-Rodríguez A. The Effectiveness of Resisted Sled

604

605 Training (RST) for Sprint Performance: A Systematic Review and Meta-analysis. Sport Med. 2018;48(9):2143-2165.

Alcaraz PE, Elvira JLL, Palao JM. Kinematic, strength, and stiffness adaptations after a short-term sled towing training in athletes. Scand J Med Sci Sports. 2014;24(2):279-290.

11. Spinks CD, Murphy AJ, Spinks WL, Lockie RG. The effects of resisted sprint training on acceleration performance and kinematics in soccer, rugby union, and Australian football players. $J$ strength Cond Res. 2007;21(1):77-85.

13. Brearley S, Bishop C. Transfer of Training. Strength Cond J. 2019;41(3):97-109.

14. Alcaraz PE, Palao JM, Elvira JLL, Linthorne NP. Effects of Three Types of Resisted Sprint Training Devices on the Kinematics of Sprinting at Maximum Velocity. J Strength Cond Res. 2008;22(3):890-897. Perform. April 2019:1-5.

16. Cotter JA, Chaudhari AM, Jamison ST, Devor ST. Knee joint kinetics in relation to commonly prescribed squat loads and depths. J strength Cond Res. 2013;27(7):1765-1774. Acceleration Performance in Team Sport Athletes. J Strength Cond Res. 2013;27(3):568-573. 
630 on: "The Effectiveness of Resisted Sled Training (RST) for Sprint Performance: A Systematic Review and 631 Meta-analysis.” Sport Med. 2019;49(2):353-356.

632 22. Atwater A. Kinematic analyses of sprinting. Track F Q Rev. 1982;82:12-16.

633 23. Haugen TA, Breitschädel F, Samozino P. Power-Force-Velocity Profiling of Sprinting Athletes. J Strength $634 \quad$ Cond Res. September 2018:1.

635

636

637

638

639

640

$641 \quad 26$

642

643

644

645

646

$647 \quad 29$.

648

649

650

651

652

653

654

655

656

657

658

659

24. Samozino P, Rabita G, Dorel S, Slawinski J, Peyrot N, Villarreal ESD, Morin JB. A simple method for measuring power, force, velocity properties, and mechanical effectiveness in sprint running. Scand J Med Sci Sports. 2016;26(26):648-658.

25. Morin JB, Samozino P, Murata M, Cross MR, Nagahara R. A simple method for computing sprint acceleration kinetics from running velocity data: Replication study with improved design. J Biomech. 2019;94:82-87.

26. Vigotsky AD, Zelik KE, Lake J, Hinrichs RN. Mechanical misconceptions: Have we lost the "mechanics" in “sports biomechanics"? J Biomech. 2019;93:1-5.

27. Morin J-B, Samozino P. Interpreting Power-Force-Velocity Profiles for Individualized and Specific Training. Int J Sports Physiol Perform. 2016;11(2):267-272.

28. Cross MR, Brughelli M, Samozino P, Brown SR, Morin J-B. Optimal Loading for Maximizing Power During Sled-Resisted Sprinting. Int J Sports Physiol Perform. 2017;12(8):1069-1077.

29. Wild JJ, Bezodis IN, North JS, Bezodis NE. Differences in step characteristics and linear kinematics between rugby players and sprinters during initial sprint acceleration. Eur J Sport Sci. July 2018:1-11.

30. von Lieres und Wilkau HC, Irwin G, Bezodis NE, Simpson S, Bezodis IN. Phase analysis in maximal sprinting: an investigation of step-to-step technical changes between the initial acceleration, transition and maximal velocity phases. Sport Biomech. July 2018:1-16.

31. Clark KP, Rieger RH, Bruno RF, Stearne DJ. The National Football League Combine 40-yd Dash. $J$ Strength Cond Res. 2019;33(6):1542-1550.

32. De Leva P. Adjustments to zatsiorsky-seluyanov’s segment inertia parameters. J Biomech. 1996;29(9):12231230 .

33. Benjamini Y, Hochberg Y. Controlling the False Discovery Rate: A Practical and Powerful Approach to Multiple Testing. J R Stat Soc Ser B. 1995;57(1):289-300.

34. Hopkins WG. A scale of magnitudes for effect statistics. A new view Stat 502.

35. Hopkins WG. Spreadsheets for Analysis of Validity and Reliability. Sportscience. 2017;21.

Peer) reviewing PDF | (2020:07:51064:2:0:NEW 5 Nov 2020) 
660 36. Bezodis NE, Salo AIT, Trewartha G. Measurement error in estimates of sprint velocity from a laser 661 displacement measurement device. Int J Sports Med. 2012;33(6):439-444.

662 37. Cottle CA, Carlson LA, Lawrence MA. Effects of Sled Towing on Sprint Starts. J Strength Cond Res. 2014;28(5):1241-1245.

664

38. Romero-Franco N, Jiménez-Reyes P, Castaño-Zambudio A, Capelo-Ramírez F, Rodríguez-Juan JJ, González-Hernández J, Toscano-Bendala FJ, Cuadrado-Peñafiel V, Balsalobre-Fernández C. Sprint performance and mechanical outputs computed with an iPhone app: Comparison with existing reference methods. Eur J Sport Sci. 2017;17(4):386-392.

668

39. Lahti J, Jiménez-Reyes P, Cross MR, Samozino P, Chassaing P, Simond-Cote B, Ahtiainen JP, Morin JB. Individual Sprint Force-Velocity Profile Adaptations to In-Season Assisted and Resisted Velocity-Based Training in Professional Rugby. Sports. 2020;8(5):74.

671

40. Schuermans J, Tiggelen D Van, Palmans T, Danneels L, Witvrouw E. Gait \& Posture Deviating running 
Figure 1

\section{Training program design}

HS: Heavy Sled, *: sled velocity verification was completed on week 1, filming of sled technique on week 2, RECO: recovery time between sprints, m: meters, FV: Force-velocity, \#: camp training included two sprints with rubber bands and $2 \times 2$ free sprints on separate days.

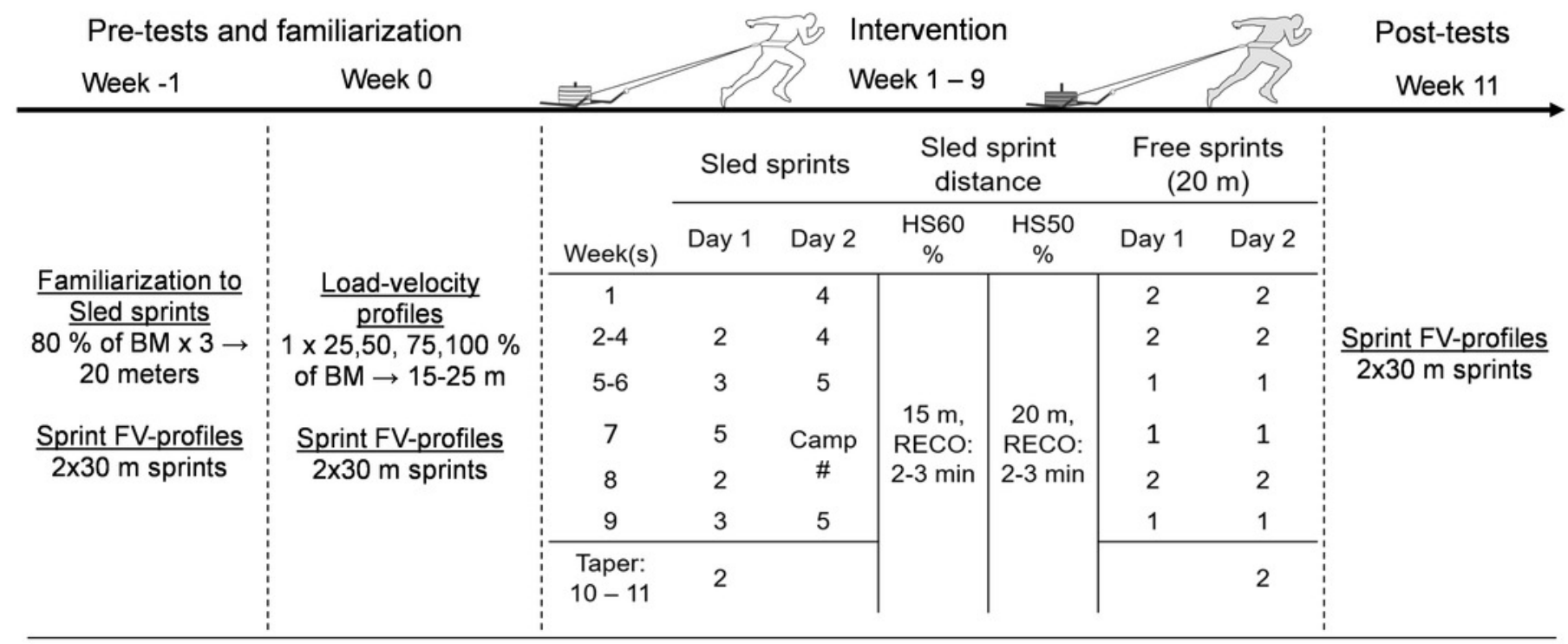




\section{Figure 2}

Sprint split-time changes.

Raw Changes in split time performance with MDC thresholds (A) and their corresponding effect sizes within each group with ES thresholds (B). The lines between the four split-time measurements $(0-5,0-10,0-20,0-30)$ have been smoothed. The error ribbons represent standard error via bias corrected and accelerated bootsrapping at 0.68 confidence intervals, corresponding to +/- 1 standard deviation. HS: Heavy sled, CON: control group, MDC: Minimal detectable change.

A)

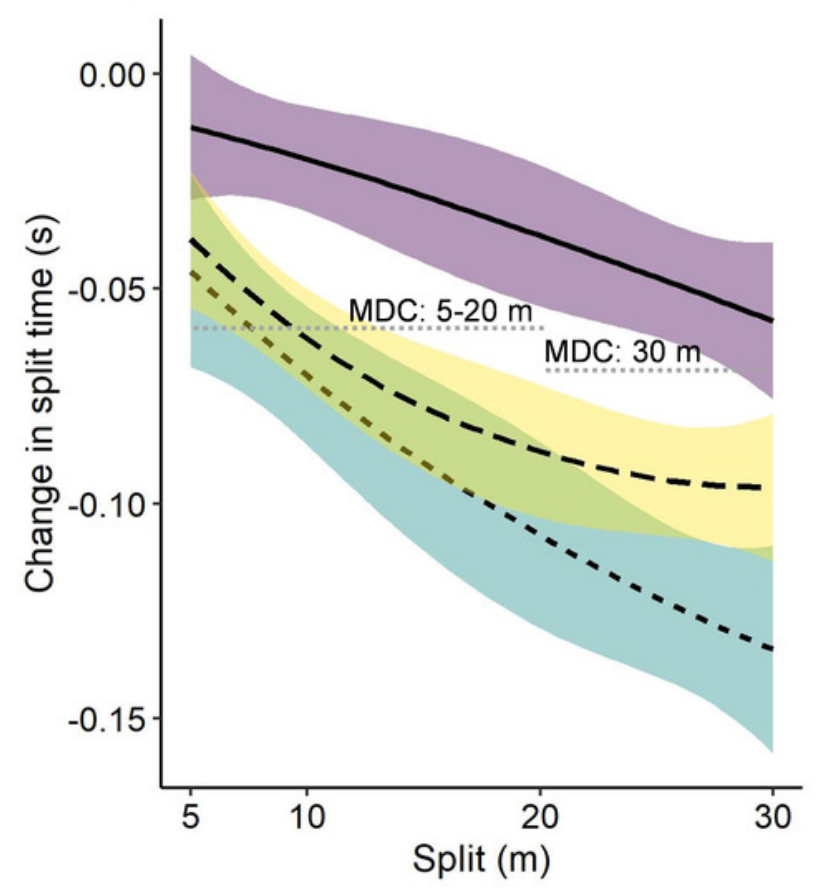

B)

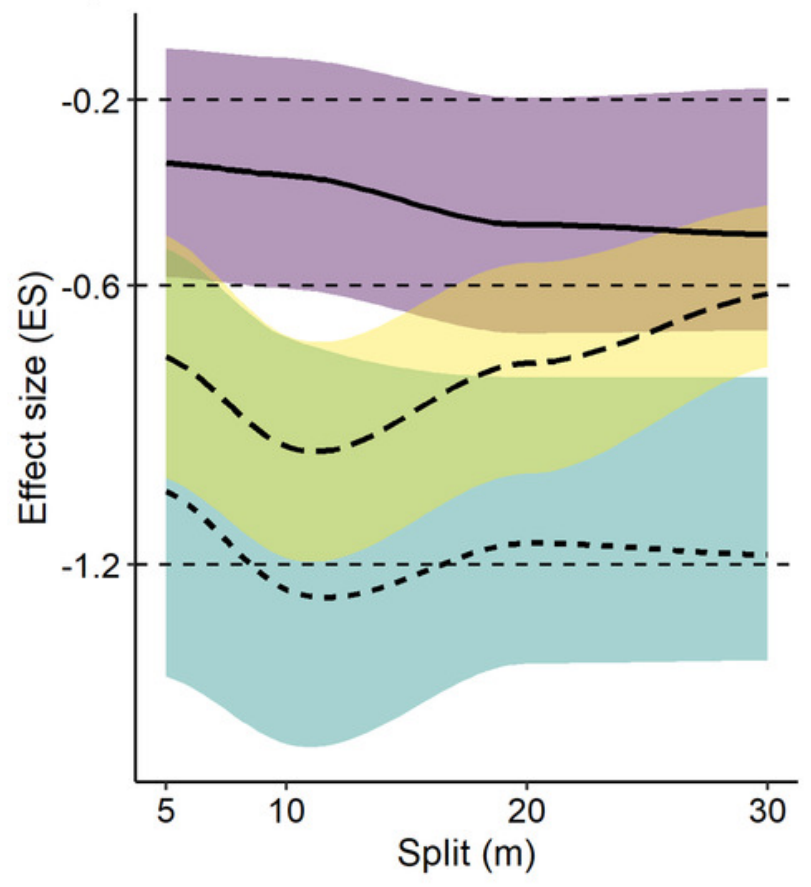


Figure 3

Mechanical variable correlations

Correlation coefficients between initial values in A) maximal theoretical horizontal force (F0) production, B) initial Sprint FV-profile (-FO/v0), and respective changes post intervention. HS: Heavy sled, CON: control group, $*: p<0.05, * *: p<0.01$.
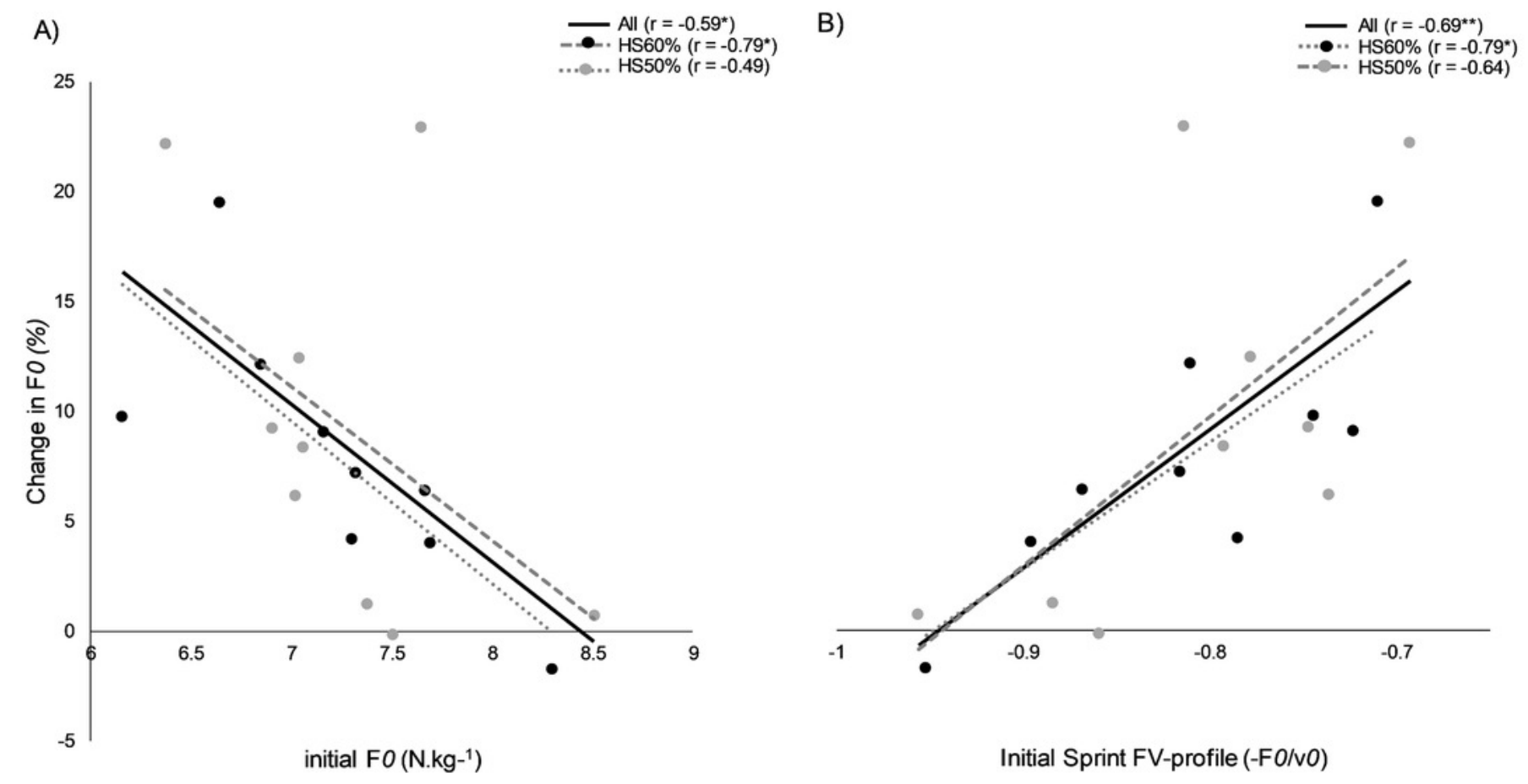


\section{Figure 4}

Sprint kinematic and spatiotemporal changes, immediate effects of sled.

Immediate kinematic and spatiotemporal differences between early acceleration (black) and sled sprinting (gray). Touchdown (A, B) and toe - off (C, D) within HS60\% and HS50\% groups. Toe-off HS: Heavy sled, CT: Contact time, SR: Step Rate, SL: Step Length relative to body height, CM: Center of Mass, IPSI: Ipsilateral (ground contact leg), m: meter, *: $p<0.05$. No group differences were found $(p<0.05)$. 
HS60\% ( $=8)$

CT: 0.19 vs $0.27 \mathrm{~s}^{*}$

SR: 4.19 vs $3.49 \mathrm{~Hz}^{*}$

SL: 0.61 vs. $0.48^{\star}$ (m/body height)
Touchdown

A)

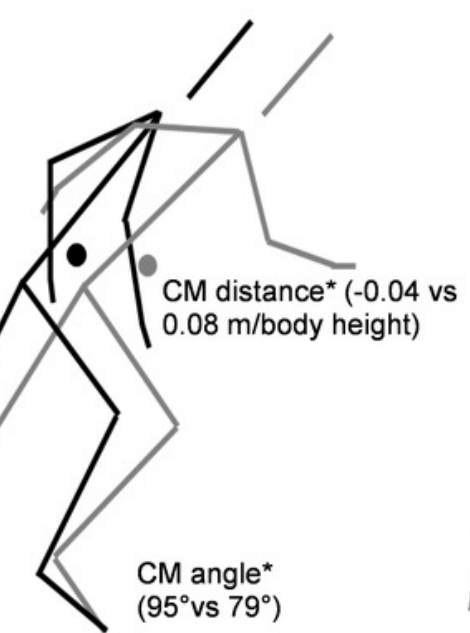

$\underline{\text { Toe - off }}$

C)

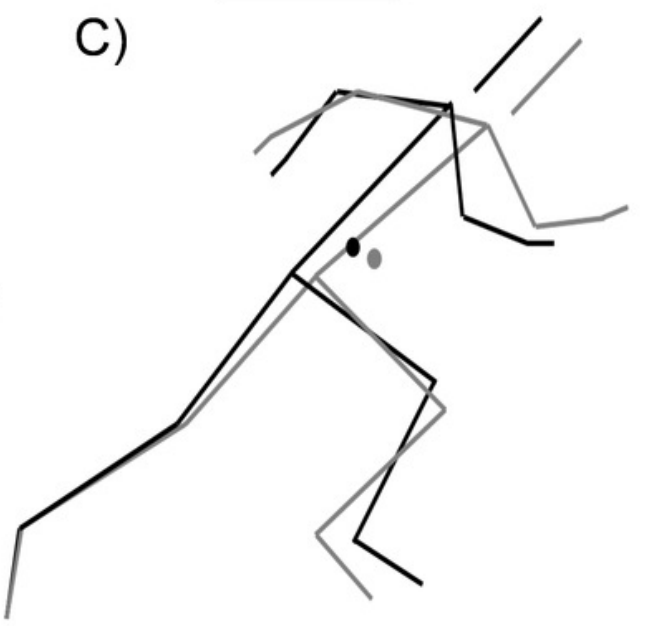

B)

$\mathrm{HS} 50 \%(\mathrm{~N}=6)$

CT: 0.19 vs $0.24 \mathrm{~s}$

SR: 4.19 vs $3.55 \mathrm{~Hz}$

SL: 0.64 vs. 0.56 (m/body height)
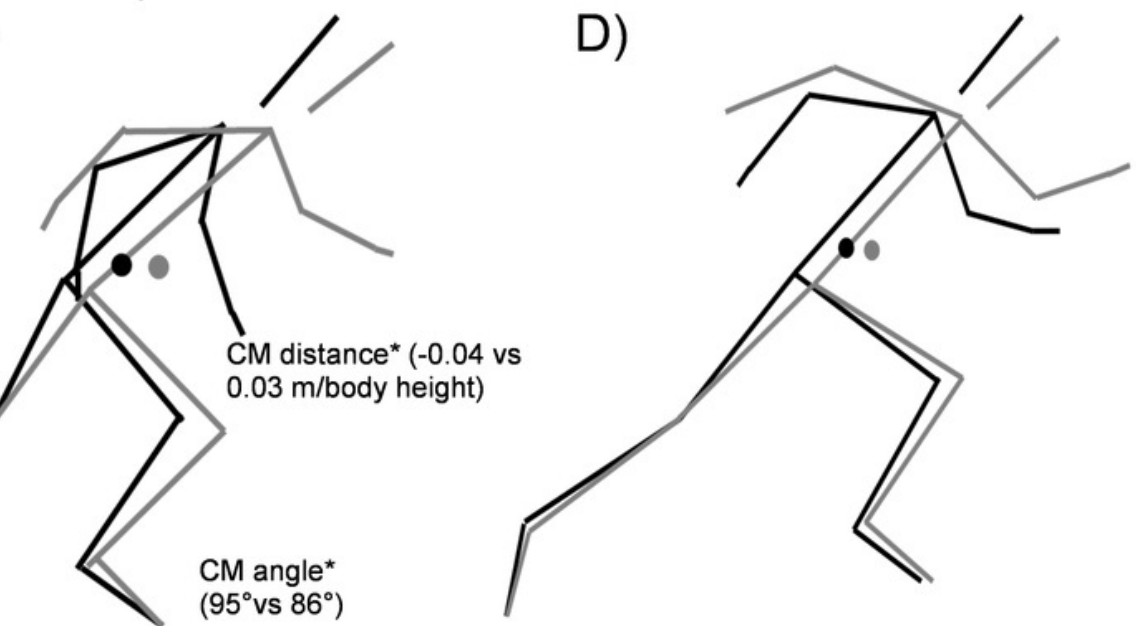

- CM early acceleration, no sled

CM immediate change with sled

Early acceleration, no sled Immediate change with sled 


\section{Figure 5}

Pre-post intervention sprint kinematic changes in early acceleration and upright sprinting.

Touchdown (A, B, C, J, I, K) and toe - off (D, E, F, H, J, L) within HS60\%, HS50\%, and CON groups. In early acceleration, toe-off is based on the average of the first push toe-off from the sprint start and the first two steps toe-off. The touchdown is based on the first 3 steps. Upright sprinting toe-off and touchdown are analyzed from 2 steps during upright sprinting at our close to maximal velocity ( $22.5 \mathrm{~m})$. No kinematic variables for within and betweengroup comparisons reached significance. Toe-off HS: Heavy sled, CT: Contact time, SR: Step rate, SL: Step Length relative to body height, CM: Center of Mass. *: Significant within-group difference $(p<0.05)$. 


\section{$\mathrm{HS} 60 \%$}

Early acceleration

CT: $0.19 \pm 0.02$ vs. $0.18 \pm 0.02 \mathrm{~s}$ SR: $4.19 \pm 0.20$ vs $4.32 \pm 0.29 \mathrm{~Hz}$ SL: $0.61 \pm 0.06$ vs. $0.62 \pm 0.06$ (m/body height)

Upright sprinting

CT: $0.13 \pm 0.01$ vs. $0.13 \pm 0.02 \mathrm{~s}$ SR: $4.30 \pm 0.25$ vs $4.48 \pm 0.19 \mathrm{~Hz}$ SL: $1.04 \pm 0.04$ vs. $1.02 \pm 0.03$ (m/body height)

\section{HS50\%}

Early acceleration

CT: $0.19 \pm 0.01$ vs. $0.19 \pm 0.03 \mathrm{~s}$ SR: $4.19 \pm 0.17$ vs $4.36 \pm 0.41 \mathrm{~Hz}$ SL: $0.63 \pm 0.04$ vs. $0.60 \pm 0.08$ ( $\mathrm{m} /$ body height)

Upright sprinting

CT: $0.12 \pm 0.01$ vs. $0.12 \pm 0.01 \mathrm{~s}$ SR: $4.47 \pm 0.12$ vs $4.65 \pm 0.12 \mathrm{~Hz}$ SL: $1.08 \pm 0.06$ vs. $1.07 \pm 0.07$ ( $\mathrm{m} /$ body height)

\section{$\mathrm{CON}$}

Early acceleration CT: $0.18 \pm 0.01$ vs. $0.18 \pm 0.01 \mathrm{~s}$ SR: $4.27 \pm 0.26$ vs $4.28 \pm 0.33 \mathrm{~Hz}$ SL: $0.62 \pm 0.05$ vs. $0.65 \pm 0.05$ (m/body height)

Upright sprinting

CT: $0.12 \pm 0.01$ vs. $0.12 \pm 0.01 \mathrm{~s}$ SR: $4.50 \pm 0.18$ vs $4.53 \pm 0.28 \mathrm{~Hz}$ SL: $1.03 \pm 0.08$ vs. $1.01 \pm 0.06$ ( $\mathrm{m} /$ body height)

\section{Touchdown}

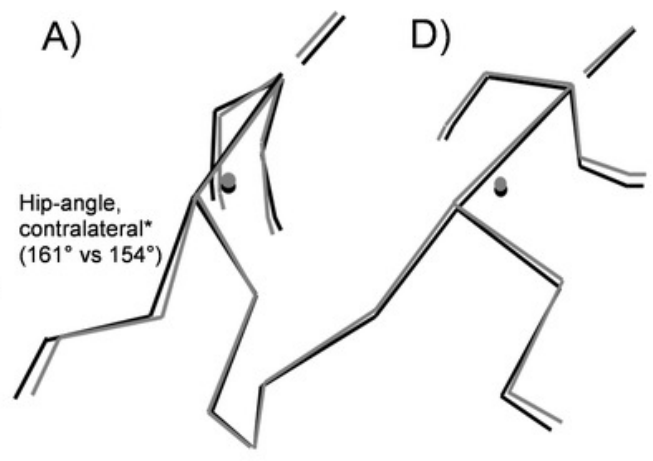

B)

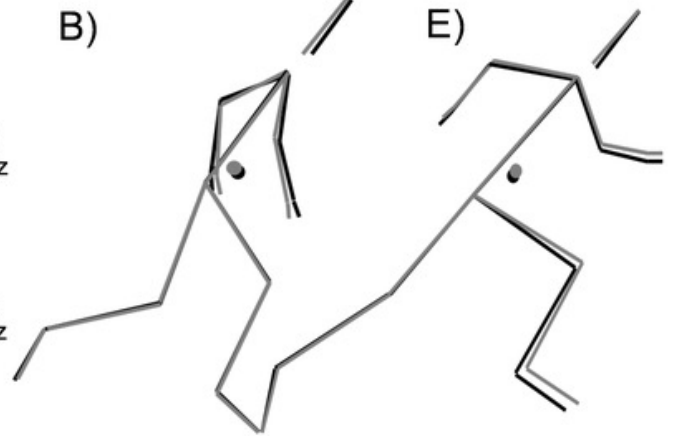

C)

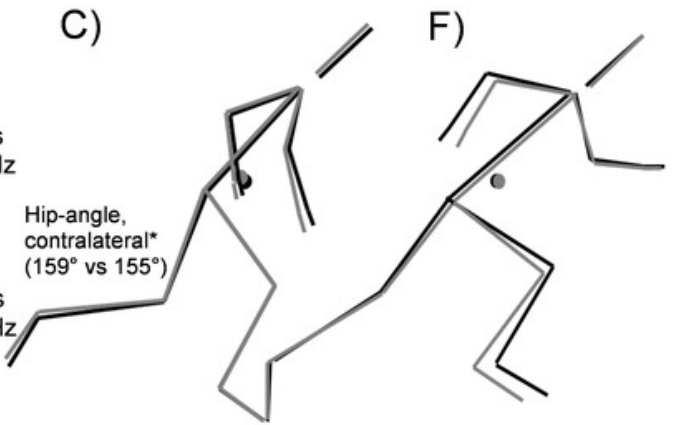

$\underline{\text { Touchdown }} \underline{\text { Toe }- \text { off }}$
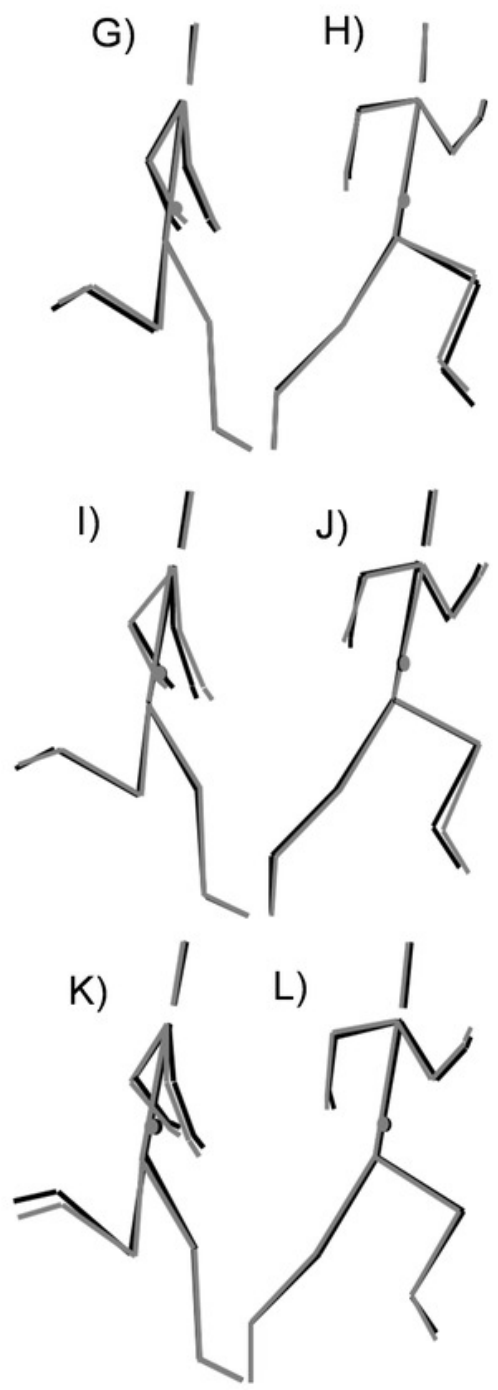


\section{Table $\mathbf{1}$ (on next page)}

Results for sprint split-times.

HS: Heavy sled, CON: Control, s: seconds, ES: Effect size (Small: $0.2-0.59$, Moderate: 0.60 1.19, Large $1.19>$ ), SD: Standard deviation, $\Delta$ : alpha (change pre post), a:Significant main effect of time, b: Significant group $\times$ time interaction effect, $*$ : Significant post-hoc difference pre- to post-intervention ( $p<.05)$, **: $(p<0.01)$, NS: Non-significant. 


\begin{tabular}{|c|c|c|c|c|c|c|c|}
\hline \multirow[b]{2}{*}{ Variable } & \multirow[b]{2}{*}{ MDC (\%) } & \multicolumn{5}{|c|}{ Within-group statistics } & \multirow{2}{*}{$\begin{array}{l}\text { Between- } \\
\text { group } \\
\text { statistics }\end{array}$} \\
\hline & & Group & Pre (SD) & Post (SD) & $\% \Delta(95 \% \mathrm{Cl})$ & $\begin{array}{c}\text { P-value (post- } \\
\text { hoc), ES }\end{array}$ & \\
\hline \multirow{3}{*}{$5 \mathrm{~m}$ split time $(\mathrm{s})^{\mathrm{a}}$} & \multirow{3}{*}{$0.06(4.00)$} & HS60\% & $1.39(0.05)$ & $1.35(0.04)$ & $-2.54(-3.56 ;-1.52)$ & $p=0.05, E S:-0.74$ & \multirow{3}{*}{ NS } \\
\hline & & $\mathrm{HS} 50 \%$ & $1.39(0.04)$ & $1.34(0.04)^{* *}$ & $-3.14(-5.63 ;-0.65)$ & $p=0.005^{* *}, E S:-1.04$ & \\
\hline & & CON & $1.38(0.04)$ & $1.36(0.04)$ & $-0.90(-2.17 ; 0.88)$ & $p=1.00, E S:-0.33$ & \\
\hline \multirow{3}{*}{$10 \mathrm{~m}$ split time $(\mathrm{s})^{\mathrm{a}, \mathrm{b}}$} & \multirow{3}{*}{$0.06(2.78)$} & HS60\% & $2.15(0.08)$ & $2.09(0.06)^{\star *}$ & $-3.05(-4.07 ;-2.03)$ & $p=0.001^{* *}, E S:-0.96$ & \multirow{3}{*}{$\begin{array}{l}\text { HS } 50 \%> \\
\text { CON, } \\
p=0.03^{*}, \text { ES: } \\
1.03\end{array}$} \\
\hline & & HS50\% & $2.14(0.06)$ & $2.07(0.06)^{\star \star}$ & $-3.37(-5.29 ;-1.46)$ & $p<0.001^{* *}, E S:-1.25$ & \\
\hline & & $\mathrm{CON}$ & $2.12(0.06)$ & $2.10(0.04)$ & $-0.87(-1.95 ;-0.52)$ & $p=0.76, E S:-0.37$ & \\
\hline \multirow{3}{*}{$20 \mathrm{~m}$ split time $(\mathrm{s})^{\mathrm{a}}$} & \multirow{3}{*}{$0.06(1.71)$} & HS60\% & $3.45(0.12)$ & $3.36(0.10)^{\star *}$ & $-2.45(-3.37 ;-1.54)$ & $p=0.008^{* *}, E S:-0.77$ & \multirow{3}{*}{ NS } \\
\hline & & HS50\% & $3.43(0.08)$ & $3.32(0.10)^{\star *}$ & $-3.07(-4.64 ;-1.51)$ & $p<0.001^{* *}, E S:-1.15$ & \\
\hline & & CON & $3.41(0.09)$ & $3.37(0.08)$ & $-1.10(-2.22 ;-0.03)$ & $p=0.44, E S:-0.47$ & \\
\hline \multirow{3}{*}{$30 \mathrm{~m}$ split time $(\mathrm{s})^{\mathrm{a}}$} & \multirow{3}{*}{$0.07(1.50)$} & HS60\% & $4.65(0.17)$ & $4.56(0.14)^{*}$ & $-2.04(-3.03 ;-1.06)$ & $p=0.021^{*}, E S:-0.62$ & \multirow{3}{*}{ NS } \\
\hline & & HS50\% & $4.62(0.10)$ & $4.49(0.12)^{\star \star}$ & $-2.89(-4.15 ;-1.64)$ & $p<0.001$, ES: -1.18 & \\
\hline & & CON & $4.62(0.12)$ & $4.56(0.11)$ & $-1.23(-2.47 ;-0.26)$ & $p=0.33, E S:-0.48$ & \\
\hline
\end{tabular}




\section{Table 2 (on next page)}

Results for sprint mechanical variables.

F0: Maximal Horizontal force, RF: Ratio of Forces, m: meters, HS: Heavy sled, CON: Control, ES: Effect size (Small: 0.2 - 0.59, Moderate: 0.60 - 1.19, Large $1.19>$ ), SD: Standard deviation, $\Delta$ : alpha (change pre post), a:Significant main effect of time, b: Significant group $x$ time interaction effect, *: Significant post-hoc difference pre- to post-intervention $(p<.05)$, **: $(p<0.01)$, NS: Non-significant. 


\begin{tabular}{|c|c|c|c|c|c|c|c|}
\hline \multirow[b]{2}{*}{ Variable } & \multirow[b]{2}{*}{ MDC \% } & \multicolumn{5}{|c|}{ Within-group statistics } & \multirow{2}{*}{$\begin{array}{c}\text { Between-group } \\
\text { statistics }\end{array}$} \\
\hline & & Group & Pre (SD) & Post (SD) & $\% \Delta(95 \% \mathrm{Cl})$ & $\begin{array}{c}\text { P-value (post- } \\
\text { hoc), ES }\end{array}$ & \\
\hline \multirow{3}{*}{$\mathrm{F} 0\left(\mathrm{~N} \cdot \mathrm{kg}^{-1}\right)^{\mathrm{a}}$} & \multirow{3}{*}{$0.68(9.53)$} & HS60\% & $7.23(0.63)$ & $7.77(0.42)^{\star}$ & $7.83(4.16 ; 11.5)$ & $\begin{array}{c}\mathrm{p}=0.018^{*}, \mathrm{ES}: \\
1.00\end{array}$ & \multirow{3}{*}{ NS } \\
\hline & & HS50\% & $7.27(0.59)$ & $7.91(0.65)^{\star \star}$ & $9.23(3.58 ; 14.9)$ & $\begin{array}{c}p=0.002^{* *}, E S: \\
1.04\end{array}$ & \\
\hline & & CON & $7.43(0.50)$ & $7.58(0.45)$ & $1.89(-1.60 ; 5.39)$ & $\begin{aligned} p= & 1.00, E S: \\
& 0.30\end{aligned}$ & \\
\hline \multirow{3}{*}{$\operatorname{RFmax}(\%)^{a}$} & \multirow{3}{*}{1.64} & HS60\% & $47.9(2.57)$ & $50.8(1.88)^{*}$ & $6.03(4.01 ; 8.03)$ & $p=\frac{0.011^{*}, E S:}{1.25}$ & \multirow{3}{*}{ NS } \\
\hline & & HS50\% & $47.9(3.51)$ & $51.2(2.91)^{\star \star}$ & $7.12(2.59 ; 11.7)$ & $p=0.001^{* *}, E S:$ & \\
\hline & & CON & $50.1(2.39)$ & $51.6(2.58)^{\star}$ & $3.00(0.42 ; 5.58)$ & $\begin{array}{c}p=0.041, E S: \\
0.55\end{array}$ & \\
\hline \multirow{3}{*}{$\begin{array}{c}\text { Mean RF } \\
\text { on 10-m (\%) }\end{array}$} & \multirow{3}{*}{4.99} & HS60\% & $27.7(1.71)$ & $28.9(1.42)^{*}$ & $4.70(2.83 ; 6.58)$ & $\begin{array}{c}p=0.013^{*}, E S: \\
0.80\end{array}$ & \multirow{3}{*}{ NS } \\
\hline & & HS50\% & $27.9(1.59)$ & $29.8(1,61)^{\star \star}$ & $6.58(4.00 ; 9.17)$ & $\begin{array}{c}\mathrm{p}<0.001, \mathrm{ES}: \\
1.14\end{array}$ & \\
\hline & & CON & $28.6(1.61)$ & $29.3(1.36)$ & $3.20(0.95 ; 5.45)$ & $p=0.05, E S: 0.65$ & \\
\hline \multirow{2}{*}{$\begin{array}{c}\text { Pmax } \\
\left(\mathrm{W} \cdot \mathrm{kg}^{-1}\right)^{\mathrm{a}, \mathrm{b}}\end{array}$} & \multirow{2}{*}{$1.10(6.97)$} & HS60\% & $16.0(1.66)$ & $17.3(1.35)^{*}$ & $8.36(5.11 ; 11.6)$ & $\begin{array}{c}\mathrm{p}=0.011^{*}, \mathrm{ES}: \\
0.84\end{array}$ & \multirow[t]{2}{*}{$\begin{array}{l}\text { HS50\% vs CON: } p= \\
0.03^{*}, \text { ES: } 1.16\end{array}$} \\
\hline & & HS50\% & $16.2(1.31)$ & $18.1(1.82)^{\star *}$ & $11.64(6.40 ; 16.9)$ & $\begin{array}{c}p<0.001, \text { ES: } \\
1.18\end{array}$ & \\
\hline
\end{tabular}




\begin{tabular}{|c|c|c|c|c|c|c|c|c|}
\hline & \multirow[b]{2}{*}{$\mathrm{CON}$} & \multirow[b]{2}{*}{$16.5(1.27)$} & \multirow[b]{2}{*}{$17.0(1.08)$} & \multirow[b]{2}{*}{$4.05(0.94 ; 7.15)$} & \multirow[b]{2}{*}{$\begin{array}{c}\mathrm{p}=0.70, \mathrm{ES}: \mathrm{:} \\
0.49\end{array}$} & \\
\hline & & & & & & & & \\
\hline 2 & \multirow{3}{*}{$\mathrm{v} 0\left(\mathrm{~m} \cdot \mathrm{s}^{-1}\right)^{\mathrm{a}}$} & \multirow{3}{*}{$0.28(3.13)$} & HS60\% & $8.93(0.51)$ & $9.08(0.39)$ & $1.79(-0.21 ; 3.78)$ & $p=1.00, E S: 0.32$ & \multirow{3}{*}{ NS } \\
\hline J & & & HS50\% & $9.03(0.36)$ & $9.31(0.33)^{*}$ & $3.08(1.44 ; 4.72)$ & $p=0.044^{\star}, \mathrm{ES}:$ & \\
\hline 4 & & & CON & $8.96(0.36)$ & $9.10(0.42)$ & $2.04(-0.45 ; 4.54)$ & $p=1.00, E S: 0.34$ & \\
\hline & \multirow{3}{*}{$\begin{array}{l}\text { Sprint FV- } \\
\text { profile } \\
(-\mathrm{FO} / \mathrm{v} 0)^{\mathrm{a}}\end{array}$} & \multirow{3}{*}{$0.06(7.37)$} & HS60\% & $-0.81(0.08)$ & $-0.86(0.05)$ & $6.07(1.54 ; 10.62)$ & $\begin{array}{c}p=0.29, \text { ES: }- \\
0.67\end{array}$ & \multirow{3}{*}{ NS } \\
\hline & & & HS50\% & $-0.81(0.08)$ & $-0.85(0.06)$ & $6.11(-0.30 ; 12.5)$ & $\begin{array}{c}p=0.57, \text { ES: }- \\
0.60\end{array}$ & \\
\hline & & & CON & $-0.83(0.07)$ & $-0.83(0.07)$ & $0.12(-5.31 ; 5.56)$ & $\begin{aligned} p= & 1.00, \text { ES: }- \\
& 0.06\end{aligned}$ & \\
\hline
\end{tabular}




\section{Table 3 (on next page)}

Results for kinematic variables from immediate effects on early acceleration of sled loads.

HS: Heavy sled, CON: control, TO: Toe-off, TD: Touchdown, CM: Center of Mass, m: meter, s: seconds, Hz: Hertz, ES: Effect size (Small: 0.2 - 0.59, Moderate: 0.60 - 1.19, Large 1.19 >), SD: Standard deviation, $\Delta$ : alpha (change pre post), *: Significant difference after controlling for multiple comparisons using the Benjamini-Hochberg procedure. 


\begin{tabular}{|c|c|c|c|c|c|c|c|c|c|}
\hline Variable & Group & $\begin{array}{c}\text { Toe-off } \\
\text { without sled }\end{array}$ & $\begin{array}{l}\text { Toe-off } \\
\text { with sled }\end{array}$ & $\% \Delta \pm \mathrm{Cl} 95 \%$ & $\begin{array}{l}\text { Within group Statistics } \\
\quad(P \text {-value, ES) }\end{array}$ & $\begin{array}{l}\text { Touchdown } \\
\text { without sled }\end{array}$ & $\begin{array}{c}\text { Touchdown with } \\
\text { sled }\end{array}$ & $\% \Delta \pm \mathrm{Cl} 195 \%$ & $\begin{array}{c}\text { Within group Statistics } \\
\text { (P-value, ES) }\end{array}$ \\
\hline \multirow{2}{*}{$\begin{array}{l}\text { CM distance } \\
\text { (m/body length) }\end{array}$} & HS60\% & $0.42(0.04)$ & $0.45(0.03)$ & $\begin{array}{c}7.74(-0.53 \\
16.0)\end{array}$ & $p=0.15, E S: 0.85$ & $-0.04(0.03)$ & $0.08(0.08)$ & $\begin{array}{c}-820(-1670 \\
29.3)\end{array}$ & $p=0.003^{*}, E S: 1.99$ \\
\hline & HS50\% & $0.43(0.01)$ & $0.46(0.03)$ & $\begin{array}{l}7.18(3.31 \\
11.0)\end{array}$ & $p=0.03, E S: 1.34$ & $-0.04(0.02)$ & $0.03(0.02)$ & $\begin{array}{c}-847(-1751 \\
55.9)\end{array}$ & $p=0.003^{*}, E S: 3.50$ \\
\hline \multirow{3}{*}{ CM angle $\left({ }^{\circ}\right)$} & HS60\% & $46.8(1.77)$ & $44.1(2.21)$ & $\begin{array}{c}-5.79(-9.90 \\
-1.67)\end{array}$ & $p=0.04, E S:-1.49$ & $95.3(4.19)$ & $79.8(8.59)$ & $\begin{array}{c}-16.1(-22.9 \\
-11.0)\end{array}$ & $p=0.005^{*}, E S:-2.30$ \\
\hline & & & & & & & & & \\
\hline & HS50\% & $46.6(1.22)$ & $44.7(1.49)$ & $\begin{array}{c}-4.46(7.41 \\
-1.52)\end{array}$ & $p=0.06, E S:-2.33$ & $95.2(3.30)$ & $86.2(2.60)$ & $\begin{array}{c}-8.46(-11.0 \\
-5.97)\end{array}$ & $p=0.005^{\star}, E S:-3.00$ \\
\hline \multirow{2}{*}{$\begin{array}{c}\text { Hip-angle } \\
\text { Ipsilateral }\left({ }^{\circ}\right)\end{array}$} & HS60\% & $171(7.61)$ & $173(10.6)$ & $\begin{array}{c}2.05(-1.91 \\
6.01)\end{array}$ & $p=0.41, E S: 0.10$ & $101(7.30)$ & $108(20.3)$ & $\begin{array}{c}7.67(-9.25 \\
24.6)\end{array}$ & $p=0.40, E S: 0.41$ \\
\hline & HS50\% & $174(2.95)$ & $181(4.82)$ & $\begin{array}{l}4.22(1.33 \\
\quad 7.11)\end{array}$ & $p=0.07, E S: 1.70$ & $105(8.10)$ & $108(4.04)$ & $\begin{array}{c}3.18(-1.40 \\
7.78)\end{array}$ & $p=0.28, E S: 0.60$ \\
\hline \multirow{2}{*}{$\begin{array}{c}\text { Hip-angle } \\
\text { Contralateral }\left({ }^{\circ}\right)\end{array}$} & HS60\% & $85.7(6.72)$ & $90.3(7.16)$ & $\begin{array}{c}6.01(-3.30 \\
15.3)\end{array}$ & $p=0.19, E S: 0.57$ & $161(8.81)$ & $159(13.1)$ & $\begin{array}{c}-0.34(-6.44 \\
5.76)\end{array}$ & $p=0.71, E S:-0.18$ \\
\hline & HS50\% & $86.7(4.08)$ & $84.7(6.09)$ & $\begin{array}{l}-3.81(-7.58 \\
-0.02)\end{array}$ & $p=0.45, E S:-0.59$ & $164(6.59)$ & $164(10.2)$ & $\begin{array}{c}2.56(-2.08 \\
7.21)\end{array}$ & $p=0.91, E S: 0.00$ \\
\hline \multirow{3}{*}{ Trunk angle $\left(^{\circ}\right)$} & HS60\% & $46.3(5.20)$ & $42.7(8.37)$ & $\begin{array}{c}-6.09(-19.0 \\
6.82)\end{array}$ & $p=0.29, E S:-0.60$ & $46.8(6.18)$ & $42.0(8.11)$ & $\begin{array}{c}-7.54(-21.2 \\
6.11)\end{array}$ & $p=0.18, E S:-0.85$ \\
\hline & & & & & & & & & \\
\hline & HS50\% & $47.9(2.87)$ & $49.4(2.76)$ & $\begin{array}{l}1.12(-4.25 \\
6.50)\end{array}$ & $p=0.31, E S: 0.33$ & $49.1(3.97)$ & $48.4(2.40)$ & $\begin{array}{c}-1.77(-6.45 \\
2.90)\end{array}$ & $p=0.66$, ES: --0.19 \\
\hline
\end{tabular}




\begin{tabular}{|c|c|c|c|c|c|}
\hline $\begin{array}{l}\text { Spatiotemporal } \\
\text { variables }\end{array}$ & Group & Early acceleration, no sled & Early acceleration, with sled & $\% \Delta \pm \mathrm{Cl} 195 \%$ & Within group Statistics (P-value, ES) \\
\hline \multirow{3}{*}{ Contact time (s) } & $\mathrm{HS} 60 \%$ & $0.191(0.02)$ & $0.274(0.05)$ & $40.0(24.5 ; 55.4)$ & $p=0.003^{*}, E S: 2.10$ \\
\hline & & & & & \\
\hline & $\mathrm{HS} 50 \%$ & $0.193(0.01)$ & $0.240(0.04)$ & $28.2(13.3 ; 43.1)$ & $p=0.03, E S: 1.71$ \\
\hline \multirow{3}{*}{ Step Rate $(\mathrm{Hz})$} & $\mathrm{HS} 60 \%$ & $4.19(0.20)$ & $3.49(0.51)$ & $-16.5(-23.3 ;-9.70)$ & $p=0.004^{*}, E S:-1.90$ \\
\hline & & & & & \\
\hline & $\mathrm{HS} 50 \%$ & $4.19(0.17)$ & $3.55(0.41)$ & $-14.8(-23.6 ;-6.12)$ & $p=0.041, E S:-2.09$ \\
\hline \multirow{2}{*}{$\begin{array}{l}\text { Step Length } \\
\text { (m/body length) }\end{array}$} & $\mathrm{HS} 60 \%$ & $0.61(0.06)$ & $0.48(0.10)$ & $-21.9(-32.3 ;-11.5)$ & $p=0.008^{*}, E S:-1.58$ \\
\hline & $\mathrm{HS} 50 \%$ & $0.64(0.04)$ & $0.56(0.04)$ & $-11.3(-16.7 ;-5.97)$ & $p=0.02, E S:-.2 .00$ \\
\hline
\end{tabular}

1 


\section{Table 4 (on next page)}

Results for kinematic and spatiotemporal variables in early acceleration (ACC) and upright sprinting (MAX).

HS: Heavy sled, CON: Control, TO: Toe-off, TD: Touchdown, CM: Center of Mass, m: meter, s:

seconds, Hz: Hertz, ES: Effect size (Small: 0.2 - 0.59, Moderate: 0.60 - 1.19, Large 1.19 >), SD: Standard deviation, $\Delta$ : alpha (change pre post). a:Significant main effect of time, *: Significant post-hoc difference pre- to post-intervention $(p<.05), * *:(p<0.01)$, NS: Nonsignificant. 


\begin{tabular}{|c|c|c|c|c|c|c|c|c|c|c|c|}
\hline \multirow[b]{2}{*}{ Variable } & \multirow{2}{*}{\multicolumn{2}{|c|}{$\begin{array}{l}\text { MDC MDC (\%) } \\
(\%) \quad \text { Touchdown } \\
\text { Toe- } \\
\text { off }\end{array}$}} & \multicolumn{9}{|c|}{ Within-group statistics } \\
\hline & & & Group & $\begin{array}{c}\text { ACC } \\
\text { Toe- } \\
\text { off } \\
\text { pre } \\
\text { (SD) }\end{array}$ & $\begin{array}{c}\text { ACC } \\
\text { Toe- } \\
\text { off } \\
\text { post } \\
\text { (SD) }\end{array}$ & $\begin{array}{c}\% \Delta \\
(95 \% \mathrm{Cl})\end{array}$ & $\begin{array}{l}\text { P-value } \\
\text { (post- } \\
\text { hoc), ES }\end{array}$ & $\begin{array}{c}\text { ACC } \\
\text { Touchdown } \\
\text { pre (SD) }\end{array}$ & $\begin{array}{c}\text { ACC } \\
\text { Touchdown } \\
\text { post (SD) }\end{array}$ & $\begin{array}{c}\% \Delta \\
(95 \% \mathrm{Cl})\end{array}$ & $\begin{array}{c}\text { P-value (post- } \\
\text { hoc), ES }\end{array}$ \\
\hline \multirow{3}{*}{$\begin{array}{l}\text { CM distance } \\
\text { m/body length }\end{array}$} & \multirow{3}{*}{$\begin{array}{c}0.04 \\
(4.76)\end{array}$} & \multirow{3}{*}{$\begin{array}{c}0.01 \\
(-55.7)\end{array}$} & HS60\% & $\begin{array}{c}0.42 \\
(0.03)\end{array}$ & $\begin{array}{c}0.42 \\
(0.04)\end{array}$ & $\begin{array}{l}-0.01(- \\
1.56 ; \\
1.36)\end{array}$ & $\begin{array}{l}p=1.00 \\
E S:-0.01\end{array}$ & $-0.04(0.03)$ & $-0.03(0.03)$ & $\begin{array}{c}39.0(-79.2 \\
157)\end{array}$ & $\begin{aligned} p= & 1.00, \text { ES: } \\
& 0.39\end{aligned}$ \\
\hline & & & HS50\% & $\begin{array}{c}0.43 \\
(0.01)\end{array}$ & $\begin{array}{c}0.43 \\
(0.01)\end{array}$ & $\begin{array}{c}0.16(- \\
1.22 ; \\
1.56)\end{array}$ & $\begin{array}{l}p=1.00 \\
E S: 0.04\end{array}$ & $-0.04(0.02)$ & $-0.02(0.03)$ & $\begin{array}{l}35.0(-420 \\
490)\end{array}$ & $\begin{aligned} p= & 0.55, \text { ES: } \\
& 0.70\end{aligned}$ \\
\hline & & & CON & $\begin{array}{c}0.43 \\
(0.02)\end{array}$ & $\begin{array}{c}0.44 \\
(0.01)\end{array}$ & $\begin{array}{l}1.04(- \\
0.82 \\
2.10)\end{array}$ & $\begin{array}{l}p=1.00 \\
E S: 0.16\end{array}$ & $-0.03(0.03)$ & $-0.03(0.02)$ & $\begin{array}{c}156(-227 \\
540)\end{array}$ & $\begin{aligned} p= & 1.00, E S: \\
& 0.00\end{aligned}$ \\
\hline \multirow{3}{*}{$\begin{array}{c}\mathrm{CM} \text { angle }\left({ }^{\circ}\right)^{\mathrm{a}} \\
\text { Relative to } \\
\text { horizontal }\end{array}$} & \multirow{3}{*}{$\begin{array}{c}1.29 \\
(2.75)\end{array}$} & \multirow{3}{*}{$\begin{array}{r}2.19 \\
(2.36)\end{array}$} & HS60\% & $\begin{array}{c}46.8 \\
(1.77)\end{array}$ & $\begin{array}{c}47.4 \\
(1.38)\end{array}$ & $\begin{array}{l}1.32(- \\
0.59 \\
3.23)\end{array}$ & $\begin{array}{l}p=1.00 \\
E S: 0.36\end{array}$ & $95.3(4.19)$ & $93.7(3.37)$ & $\begin{array}{c}-1.63(-3.02 ;- \\
0.25)\end{array}$ & $\begin{array}{c}p=1,00, E S:- \\
0.42\end{array}$ \\
\hline & & & HS50\% & $\begin{array}{c}46.6 \\
(1.22)\end{array}$ & $\begin{array}{c}46.8 \\
(1.08)\end{array}$ & $\begin{array}{l}0.46(- \\
0.64 \\
1.57)\end{array}$ & $\begin{array}{l}p=1.00 \\
E S: 0.17\end{array}$ & $95.2(3.30)$ & $92.6(4.18)$ & $\begin{array}{c}-2.66(-6.16 \\
0.82)\end{array}$ & $\begin{aligned} p= & 0.46, \text { ES: }- \\
& 0.69\end{aligned}$ \\
\hline & & & CON & $\begin{array}{c}47.7 \\
(1.97)\end{array}$ & $\begin{array}{c}47.5 \\
(1.24)\end{array}$ & $\begin{array}{l}0.45(- \\
0.81 \\
1.71)\end{array}$ & $\begin{array}{l}p=1.00 \\
E S: 0.11\end{array}$ & 93.7 (4.99) & $93.3(3.13)$ & $\begin{array}{c}-0.32(-2.36 \\
1.72)\end{array}$ & $\begin{array}{c}\mathrm{p}=1.00, \mathrm{ES}:- \\
0.10\end{array}$ \\
\hline $\begin{array}{c}\text { Hip-angle } \\
\text { Ipsilateral }\left({ }^{\circ}\right)\end{array}$ & & & HS60\% & $\begin{array}{c}171 \\
(7.61)\end{array}$ & $\begin{array}{c}169 \\
(6.72)\end{array}$ & $\begin{array}{c}-1.19(- \\
3.07 \\
0.68)\end{array}$ & $\begin{array}{l}p=0.72 \\
E S:-0.30\end{array}$ & $101(7.30)$ & $103(5.28)$ & $\begin{array}{l}1.94(-2.25 \\
6.14)\end{array}$ & $\begin{array}{c}p=1.00, E S: \\
0.26\end{array}$ \\
\hline $180^{\circ}=$ full EXT & & & & & & & & & & & \\
\hline
\end{tabular}




\begin{tabular}{|c|c|c|c|c|c|c|c|c|c|c|c|}
\hline & $\begin{array}{c}6.31 \\
(3.73)\end{array}$ & $\begin{array}{c}10.7 \\
(10.2)\end{array}$ & HS50\% & $\begin{array}{l}174 \\
(2.95)\end{array}$ & $\begin{array}{c}175 \\
(2.69)\end{array}$ & $\begin{array}{c}0.12(- \\
1.59 \\
1.82)\end{array}$ & $\begin{array}{l}p=1.00 \\
\text { ES: } 0.05\end{array}$ & $104(8.10)$ & $105(6.14)$ & $\begin{array}{c}0.74(-3.27 \\
\quad 4.75)\end{array}$ & $\begin{aligned} p= & 1.00, E S: \\
& 0.07\end{aligned}$ \\
\hline & & & CON & $\begin{array}{l}170 \\
(5.28)\end{array}$ & $\begin{array}{c}171 \\
(3.18)\end{array}$ & $\begin{array}{c}0.41(- \\
0.51 \\
1.33)\end{array}$ & $\begin{array}{l}p=1.00 \\
\text { ES: } 0.14\end{array}$ & $103(8.73)$ & $103(5.95)$ & $\begin{array}{l}1.22(-2.01 \\
4.44)\end{array}$ & $\begin{aligned} p= & 1.00, E S: \\
& 0.12\end{aligned}$ \\
\hline \multirow{3}{*}{$\begin{array}{c}\text { Hip-angle } \\
\text { Contralateral }\left({ }^{\circ}\right)^{\text {a }} \\
180^{\circ}=\text { full EXT }\end{array}$} & \multirow{3}{*}{$\begin{array}{c}5.97 \\
(7.11)\end{array}$} & \multirow{3}{*}{$\begin{array}{c}9.12 \\
(5.85)\end{array}$} & HS60\% & $\begin{array}{l}85.7 \\
(6.72\end{array}$ & $\begin{array}{l}82.8 \\
(3.98)\end{array}$ & $\begin{array}{c}-3.03(- \\
5.91 ;- \\
0.15)\end{array}$ & $\begin{array}{l}p=0.33 \\
\text { ES: }-0.51\end{array}$ & $161(8.81)$ & 154 (7.49) & $\begin{array}{c}-4.01(-5.97 ;- \\
2.05)\end{array}$ & $\begin{array}{c}p=0.004^{\star *}, \text { ES: }- \\
0.80\end{array}$ \\
\hline & & & HS50\% & $\begin{array}{l}86.7 \\
(4.08)\end{array}$ & $\begin{array}{l}85.6 \\
(5.74)\end{array}$ & $\begin{array}{c}-1.25(- \\
4.62 ; \\
2.10)\end{array}$ & $\begin{array}{l}p=1.00 \\
\text { ES: }-0.22\end{array}$ & $164(6.59)$ & $162(4.87)$ & $\begin{array}{c}-1.57(-4.68 \\
1.56)\end{array}$ & $\begin{aligned} p= & 1.00, E S:- \\
& 0.48\end{aligned}$ \\
\hline & & & CON & $\begin{array}{l}85.1 \\
(8.98)\end{array}$ & $\begin{array}{c}84.6 \\
(8.04)\end{array}$ & $\begin{array}{c}-0.47(- \\
2.39 ; \\
1.46)\end{array}$ & $\begin{array}{l}p=1.00 \\
\text { ES: }-0.06\end{array}$ & $159(7.18)$ & $155(5.36)$ & $\begin{array}{c}-3.13(-4.65 ;- \\
1.61)\end{array}$ & $\begin{array}{c}p=0.006^{* *}, E S:- \\
0.80\end{array}$ \\
\hline \multirow{3}{*}{$\begin{array}{c}\text { Trunk angle }\left({ }^{\circ}\right) \\
\text { Relative to } \\
\text { horizontal }\end{array}$} & \multirow{3}{*}{$\begin{array}{c}4.97 \\
(10.8)\end{array}$} & \multirow{3}{*}{$\begin{array}{c}6.62 \\
(14.2)\end{array}$} & $\mathrm{HS} 60 \%$ & $\begin{array}{l}46.3 \\
(5.20)\end{array}$ & $\begin{array}{l}45.3 \\
(3.03)\end{array}$ & $\begin{array}{c}-1.48(- \\
6.44 \\
3.47)\end{array}$ & $\begin{array}{l}p=1.00 \\
\text { ES: }-0.23\end{array}$ & $46.8(6.18)$ & $45.9(2.59)$ & $\begin{array}{c}-0.73(-7.25 \\
5.79)\end{array}$ & $\begin{aligned} p= & 1.00, \text { ES: }- \\
& 0.18\end{aligned}$ \\
\hline & & & HS50\% & $\begin{array}{l}47.9 \\
(2.87)\end{array}$ & $\begin{array}{c}48.6 \\
(3.77)\end{array}$ & $\begin{array}{l}1.44(- \\
2.54 \\
5.41)\end{array}$ & $\begin{array}{l}p=1.00 \\
\text { ES: } 0.20\end{array}$ & $49.1(3.97)$ & $48.8(4.25)$ & $\begin{array}{c}-0.39(-4.50 \\
4.21)\end{array}$ & $\begin{aligned} p= & 1.00, \text { ES: }- \\
& 0.07\end{aligned}$ \\
\hline & & & $\mathrm{CON}$ & $\begin{array}{l}46.5 \\
(5.29)\end{array}$ & $\begin{array}{l}46.6 \\
(4.29)\end{array}$ & $\begin{array}{c}0.59(- \\
2.10 \\
3.28)\end{array}$ & $\begin{array}{l}p=1.00 \\
\text { ES: } 0.03\end{array}$ & $47.3(5.50)$ & $46.0(4.24)$ & $\begin{array}{c}-2.26(-6.25 \\
1.73)\end{array}$ & $\begin{aligned} p= & 1.00, \text { ES: }- \\
& 0.26\end{aligned}$ \\
\hline $\begin{array}{l}\text { Spatiotemporel } \\
\text { variables ACC }\end{array}$ & \multicolumn{2}{|c|}{ MDC (\%) } & Group & \multicolumn{2}{|c|}{ Pre (SD) } & \multicolumn{2}{|c|}{ Post (SD) } & \multicolumn{2}{|c|}{$\% \Delta(95 \% \mathrm{Cl})$} & \multicolumn{2}{|c|}{ P-value (post-hoc), ES } \\
\hline \multirow{2}{*}{ Contact time (s) } & \multicolumn{2}{|c|}{0.02} & HS60\% & \multicolumn{2}{|c|}{$0.19(0.02)$} & \multicolumn{2}{|c|}{$0.18(0.02)$} & \multicolumn{2}{|c|}{$-5.48(-9.12 ;-1.83)$} & \multicolumn{2}{|c|}{$p=1.00, E S: 0.56$} \\
\hline & \multicolumn{2}{|c|}{$(9.32)$} & HS50\% & \multicolumn{2}{|c|}{$0.19(0.01)$} & \multicolumn{2}{|c|}{$0.19(0.03)$} & \multicolumn{2}{|c|}{$-0.97(-13.0 ; 11.01)$} & \multicolumn{2}{|c|}{$p=1.00, E S:-0.12$} \\
\hline
\end{tabular}




\begin{tabular}{|c|c|c|c|c|c|c|c|c|c|c|}
\hline & & & CON & $0.19(0.01)$ & \multicolumn{2}{|c|}{$0.18(0.01)$} & \multicolumn{2}{|c|}{$-2.34(-6.50 ; 1.82)$} & \multicolumn{2}{|c|}{$p=1.00$, ES: -0.34} \\
\hline \multirow{3}{*}{ Step Rate $(\mathrm{Hz})$} & \multirow{3}{*}{\multicolumn{2}{|c|}{$\begin{array}{l}0.25 \\
(5.71)\end{array}$}} & $\mathrm{HS} 60 \%$ & $4.19(0.20)$ & \multicolumn{2}{|c|}{$4.32(0.29)$} & \multicolumn{2}{|c|}{$3.25(-0.56 ; 7.07)$} & \multicolumn{2}{|c|}{$p=1.00, E S: 0.54$} \\
\hline & & & HS50\% & $4.19(0.17)$ & \multicolumn{2}{|c|}{$4.36(0.41)$} & \multicolumn{2}{|c|}{$4.45(-3.09 ; 12.0)$} & \multicolumn{2}{|c|}{$p=1.00, E S: 0.56$} \\
\hline & & & CON & $4.27(0.26)$ & \multicolumn{2}{|c|}{$4.28(0.33)$} & \multicolumn{2}{|c|}{$0.54(-2.61 ; 3.69)$} & \multicolumn{2}{|c|}{$p=1.00, E S: 0.08$} \\
\hline \multirow{3}{*}{$\begin{array}{l}\text { Step Length } \\
\text { (m/body length })^{a}\end{array}$} & \multirow{3}{*}{\multicolumn{2}{|c|}{$\begin{array}{l}0.05 \\
(4.89)\end{array}$}} & $\mathrm{HS} 60 \%$ & $0.61(0.06)$ & \multicolumn{2}{|c|}{$0.62(0.06)$} & \multicolumn{2}{|c|}{$1.52(-3.21 ; 6.24)$} & \multicolumn{2}{|c|}{$p=1.00, E S: 0.13$} \\
\hline & & & HS50\% & $0.64(0.03)$ & \multicolumn{2}{|c|}{$0.64(0.04)$} & \multicolumn{2}{|c|}{$0.15(-2.96 ; 3.26)$} & \multicolumn{2}{|c|}{$p=1.00, E S:-0.50$} \\
\hline & & & CON & $0.62(0.05)$ & \multicolumn{2}{|c|}{$0.65(0.05)$} & \multicolumn{2}{|c|}{$5.38(1.12 ; 9.64)$} & \multicolumn{2}{|c|}{$p=0.23, E S: 0.60$} \\
\hline Variable & $\begin{array}{l}\text { MDC } \\
(\%) \\
\text { Toe- } \\
\text { off }\end{array}$ & $\begin{array}{l}\text { MDC }(\%) \\
\text { Touch- } \\
\text { down }\end{array}$ & Group & $\begin{array}{cc}\text { MAX } & \text { MAX } \\
& \text { Toe-off } \\
\text { Toe-off } & \text { post } \\
\text { pre (SD) } & (S D)\end{array}$ & $\begin{array}{c}\% \Delta \\
(95 \% \mathrm{Cl})\end{array}$ & $\begin{array}{c}\text { P-value } \\
\text { (post-hoc), } \\
\text { ES }\end{array}$ & $\begin{array}{c}\text { MAX } \\
\text { Touchdown pre (SD) }\end{array}$ & $\begin{array}{c}\text { MAX } \\
\text { Touchdown } \\
\text { post (SD) }\end{array}$ & $\begin{array}{c}\% \Delta \\
(95 \% \mathrm{Cl})\end{array}$ & $\begin{array}{l}\text { P-value (post- } \\
\text { hoc), ES }\end{array}$ \\
\hline \multirow{3}{*}{$\begin{array}{l}\mathrm{CM} \text { distance to toe } \\
\mathrm{m} / \text { body length }\end{array}$} & \multirow{3}{*}{$\begin{array}{l}0.05 \\
(8.27)\end{array}$} & \multirow{3}{*}{$\begin{array}{c}0.04 \\
(-12.1)\end{array}$} & $\mathrm{HS} 60 \%$ & $0.35(0.01) 0.34(0.01)$ & $\begin{array}{c}-2.09(-3.76 ;- \\
0.41)\end{array}$ & $\begin{aligned} & p=1.00, E S:- \\
& 0.48\end{aligned}$ & $-0.23(0.02)$ & $-0.21(0.02)$ & $-5.84(-10.9 ;-0.83)$ & $p=0.63, E S: 0.71$ \\
\hline & & & HS50\% & $0.34(0.02) 0.36(0.03)$ & $\begin{array}{c}3.67(-1.51 \\
8.85)\end{array}$ & $\begin{aligned} p= & 0.63, E S: \\
& 0.44\end{aligned}$ & $-0.22(0.02)$ & $-0.21(0.01)$ & $-2.81(-6.77 ; 1.16)$ & $p=1.00, E S: 0.44$ \\
\hline & & & CON & $0.33(0.02) 0.33(0.02)$ & $\begin{array}{l}-0.19(-1.57 ; \\
\quad 1.19)\end{array}$ & $\begin{aligned} p= & 1.00, E S:- \\
& 0.02\end{aligned}$ & $-0.21(0.02)$ & $-0.21(0.02)$ & $-1.11(-4.75 ; 2.53)$ & $p=1.00, E S: 0.09$ \\
\hline \multirow{3}{*}{$\mathrm{CM}$ angle $\left({ }^{\circ}\right)^{\mathrm{a}}$} & \multirow{3}{*}{$\begin{array}{l}2.21 \\
(3.87)\end{array}$} & \multirow{3}{*}{$\begin{array}{l}2.94 \\
(2.64)\end{array}$} & $\mathrm{HS} 60 \%$ & $56.6(2.13) 57.1(1.87)$ & $\begin{array}{l}0.95(0.19 \\
1.71)\end{array}$ & $\begin{aligned} p= & 1.00, E S: \\
& 0.26\end{aligned}$ & $114(2.11)$ & $112(2.11)$ & $-1.23(-2.27 ;-0.20)$ & $p=0.50, E S:-0.67$ \\
\hline & & & HS50\% & $57.6(2.77) 56.1(2.63)$ & $\begin{array}{c}-2.48(0.19 \\
0.44)\end{array}$ & $\begin{array}{c}p=0.55, \text { ES: - } \\
0.54\end{array}$ & $112(1.64)$ & $112(2.01)$ & $-0.44(-1.16 ; 0.28)$ & $p=1.00, E S:-0.27$ \\
\hline & & & CON & $56.4(2.38) 57.7(2.17)$ & $\begin{array}{l}2.40(0.77 \\
4.03)\end{array}$ & $\begin{aligned} & p= 0.32, E S: \\
& 0.58\end{aligned}$ & $112(2.37)$ & $112(2.49)$ & $0.03(-0.83 ; 0.90)$ & $p=1.00, E S: 0.01$ \\
\hline
\end{tabular}




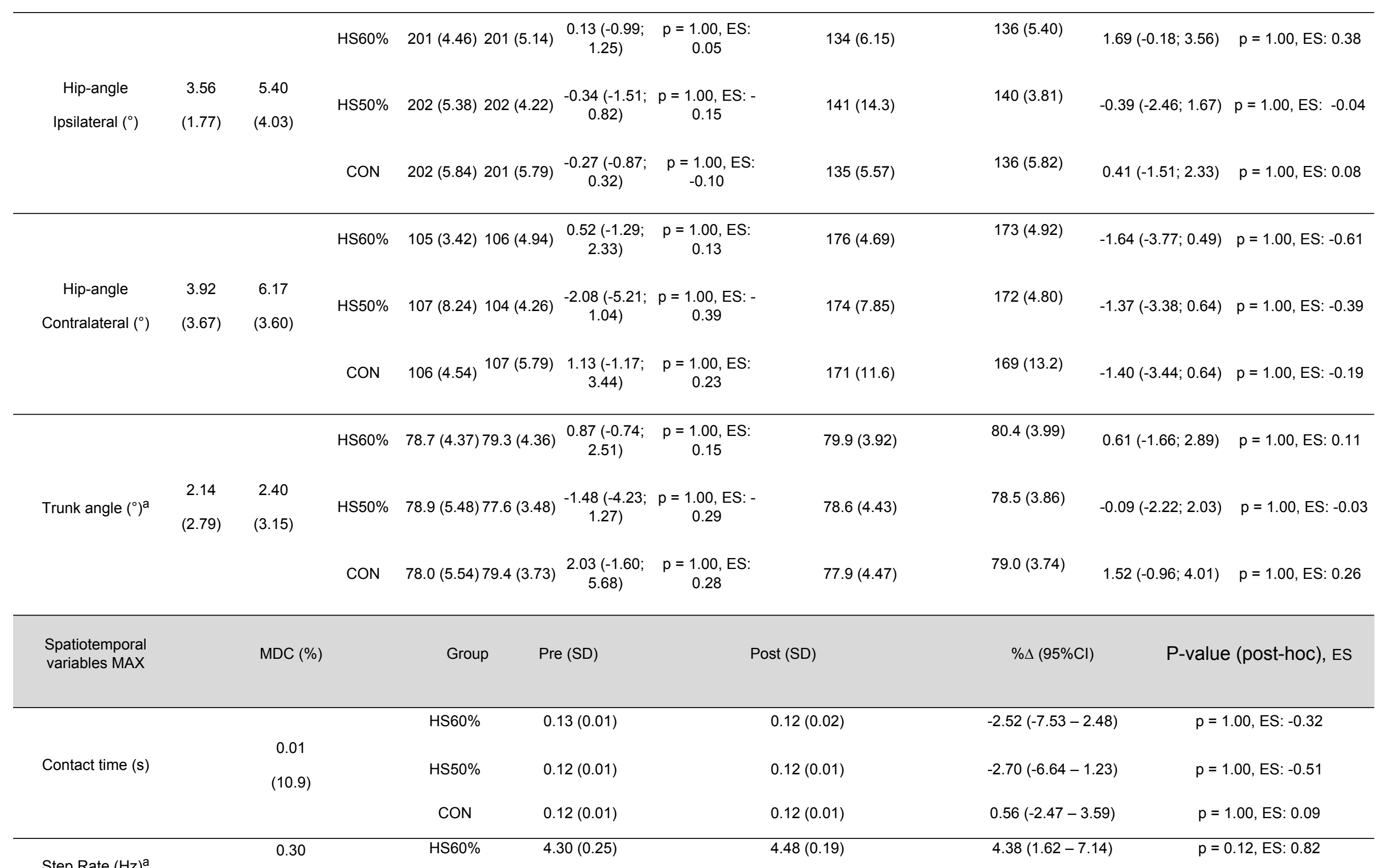




\begin{tabular}{|c|c|c|c|c|c|c|}
\hline & \multirow[t]{2}{*}{$(6.60)$} & HS50\% & $4.47(0.12)$ & $4.65(0.12)$ & $4.00(1.66-6.33)$ & $p=0.90, E S: 1.50$ \\
\hline & & CON & $4.50(0.18)$ & $4.53(0.28)$ & $0.67(-2.82-4.17)$ & $p=1.00, E S: 0.12$ \\
\hline \multirow{3}{*}{$\begin{array}{l}\text { Step Length } \\
\text { m/body length }\end{array}$} & \multirow{3}{*}{$\begin{array}{l}0.08 \\
(4.53)\end{array}$} & HS60\% & $1.04(0.04)$ & $1.02(0.03)$ & $-1.39(-3.07-0.28)$ & $p=1.00, E S:-0.39$ \\
\hline & & HS50\% & $1.08(0.06)$ & $1.07(0.07)$ & $-1.37(-3.75-1.00)$ & $p=1.00, E S:-0.23$ \\
\hline & & $\mathrm{CON}$ & $1.03(0.08)$ & $1.01(0.06)$ & $-1.38(-5.26-2.50)$ & $p=1.00$, ES: -0.23 \\
\hline
\end{tabular}

1 